\title{
EQUIVARIANT K-THEORY OF GENERALIZED STEINBERG VARIETIES
}

\author{
J. MATTHEW DOUGLASS AND GERHARD RÖHRLE
}

\begin{abstract}
We describe the equivariant $K$-groups of a family of generalized Steinberg varieties that interpolates between the Steinberg variety of a reductive, complex algebraic group and its nilpotent cone in terms of the extended affine Hecke algebra and double cosets in the extended affine Weyl group. As an application, we use this description to define Kazhdan-Lusztig "bar" involutions and Kazhdan-Lusztig bases for these equivariant $K$-groups.
\end{abstract}

\section{InTRODUCTION}

Suppose $G\left(\mathbb{F}_{q}\right)$ is a Chevalley group defined over the finite field $\mathbb{F}_{q}$. A fundamental result in the classification of irreducible complex representations of $G\left(\mathbb{F}_{q}\right)$ is the classification of representations that contain a vector fixed by a Borel subgroup $B\left(\mathbb{F}_{q}\right)$ of $G\left(\mathbb{F}_{q}\right)$. These representations are completely determined, and their characters may be computed, using the centralizer ring or Hecke algebra $\mathcal{H}\left(G\left(\mathbb{F}_{q}\right), B\left(\mathbb{F}_{q}\right)\right)$. Iwahori [10] conjectured that $\mathcal{H}\left(G\left(\mathbb{F}_{q}\right), B\left(\mathbb{F}_{q}\right)\right)$ is isomorphic to the group algebra of the Weyl group $W$ of $G\left(\mathbb{F}_{q}\right)$. An explicit isomorphism between $\mathcal{H}\left(G\left(\mathbb{F}_{q}\right), B\left(\mathbb{F}_{q}\right)\right.$ ) and the group algebra of $W$ was constructed by Lusztig [13]. More generally, irreducible representations that contain a vector fixed by a parabolic subgroup $P_{I}\left(\mathbb{F}_{q}\right)$ of $G\left(\mathbb{F}_{q}\right)$ are determined by the parabolic Hecke algebra $\mathcal{H}\left(G\left(\mathbb{F}_{q}\right), P_{I}\left(\mathbb{F}_{q}\right)\right)$. Curtis, Iwahori, and Kilmoyer [4] showed that this last algebra is isomorphic to the Hecke algebra $\mathcal{H}\left(W, W_{I}\right)$, where $W_{I}$ is the corresponding parabolic subgroup of $W$, and Curtis [3] extended Lusztig's construction to obtain an explicit isomorphism between $\mathcal{H}\left(G\left(\mathbb{F}_{q}\right), P_{I}\left(\mathbb{F}_{q}\right)\right)$ and $\mathcal{H}\left(W, W_{I}\right)$.

Now suppose $G\left(\mathbb{Q}_{p}\right)$ is a Chevalley group defined over $\mathbb{Q}_{p}$. In this case, one important class of representations consists of those representations that contain a vector fixed by an Iwahori subgroup of $G\left(\mathbb{Q}_{p}\right)$. These representations are again classified by a Hecke algebra, this time the extended affine Hecke algebra $\mathcal{H}$ of the complex dual group, $\breve{G}$. Kazhdan and Lusztig [12] construct an isomorphism between $\mathcal{H}$ and the equivariant $K$-theory of the Steinberg variety of $\check{G}$. They then use this isomorphism to give a construction of the irreducible representations of $\mathcal{H}$. In this paper we extend their construction and explicitly describe the equivariant $K$-groups of the generalized Steinberg varieties $X^{I J}$ from [7] in terms of the Hecke algebra $\mathcal{H}$. When $I=J$, the subspace of $\mathcal{H}$ we consider is, up to an involution, the extension to $\mathcal{H}$ of the subalgebra $\mathcal{H}\left(G\left(\mathbb{F}_{q}\right), P_{I}\left(\mathbb{F}_{q}\right)\right)$ of $\mathcal{H}\left(G\left(\mathbb{F}_{q}\right), B\left(\mathbb{F}_{q}\right)\right)$.

2010 Mathematics Subject Classification. Primary 20G05; Secondary 14L30 20 C08.

Key words and phrases. Equivariant $K$-theory, Hecke algebra, Steinberg variety. 
In another direction, it follows from [8, Theorem 5.1.3] and [9, Theorem 2.5] that the rational Borel-Moore homology of $X^{I J}$ may be computed algebraically as the space of $W_{I} \times W_{J}$-invariants in the smash (semidirect) product of the coinvariant algebra of $W$ with the group algebra of $W$. The results in this paper may be viewed as the extension of this computation to the more refined level of equivariant $K$-theory and the affine Hecke algebra.

From now on, suppose that $G$ is a connected, reductive complex algebraic group such that the derived group of $G$ is simply connected. Set $\mathfrak{g}=\operatorname{Lie}(G)$. For $g \in G$ and $x \in \mathfrak{g}$ write $g x$ instead of $\operatorname{Ad}(g)(x)$, where Ad is the adjoint representation of $G$. Define $\mathfrak{N}$ to be the cone of nilpotent elements in $\mathfrak{g}$ and let $B$ be a fixed Borel subgroup of $G$ with Lie algebra $\mathfrak{b}$. Then, the Steinberg variety of $G$ is the variety $Z$ of all triples $(x, g B, h B)$ in $\mathfrak{N} \times G / B \times G / B$ such that $g^{-1} x, h^{-1} x \in \mathfrak{b}$. Based on a construction of Kazhdan and Lusztig [12], Chriss and Ginzburg [2] and Lusztig [15] have shown that there is an algebra structure on $K^{G \times \mathbb{C}^{*}}(Z)$, the $G \times \mathbb{C}^{*}$-equivariant $K$-group of $Z$, such that $K^{G \times \mathbb{C}^{*}}(Z)$ is isomorphic to the extended, affine Hecke algebra $\mathcal{H}$ associated to $G$. Ostrik [17] used this isomorphism to describe $K^{G \times \mathbb{C}^{*}}(\mathfrak{N})$ in terms of $\mathcal{H}$ and to define a Kazhdan-Lusztig "bar" involution, and a Kazhdan-Lusztig basis, of $K^{G \times \mathbb{C}^{*}}(\mathfrak{N})$. As indicated above, in this paper we describe the equivariant $K$-groups of the generalized Steinberg varieties $X^{I J}$ in terms of $\mathcal{H}$. These generalized Steinberg varieties interpolate between $Z=X^{\emptyset \emptyset}$ and $\mathfrak{N}=X^{S S}$ ( $S$ is the Coxeter generating set for $W$ determined by $B$ ). We then use our description to define Kazhdan-Lusztig "bar" involutions and Kazhdan-Lusztig bases of the equivariant $K$-groups $K^{G \times \mathbb{C}^{*}}\left(X^{I J}\right)$.

The proof of the main theorem in this paper (Theorem 2.1) relies on Ostrik's computation of $K^{G \times \mathbb{C}^{*}}(\mathfrak{N})$. For a generalized Steinberg variety $X^{I J}$ we use a filtration of $K^{G \times \mathbb{C}^{*}}\left(X^{I J}\right)$ indexed by $G$-orbits in the product of two partial flag varieties. In the special case of $\mathfrak{N}$, there is a single $G$-orbit, the filtration of $K^{G \times \mathbb{C}^{*}}(\mathfrak{N})$ is trivial, and Ostrik has computed $K^{G \times \mathbb{C}^{*}}(\mathfrak{N})$ in terms of $\mathcal{H}$. In the general case, each associated graded piece has the form $K^{L^{\prime} \times \mathbb{C}^{*}}\left(\mathfrak{N}^{\prime}\right)$, where $L^{\prime}$ is a Levi factor of a parabolic subgroup of $G$ and $\mathfrak{N}^{\prime}$ is the nilpotent cone in $\operatorname{Lie}\left(L^{\prime}\right)$. Thus, each of these graded pieces is described using Ostrik's theory.

In $\S 2$ we give the basic constructions and state the main theorem relating the extended affine Hecke algebra and the equivariant $K$-theory of generalized Steinberg varieties. Assuming facts that are proved in subsequent sections, $\S 3$ contains a proof of Theorem 2.1. The constructions of the "standard basis," the "bar" involution, and the Kazhdan-Lusztig basis are given in $\S 4$. In $\S 5$ we review the intersection/Tor product construction in the form it is used in this paper. The final three sections contain proofs of the main ingredients used in the proof of Theorem 2.1.

\subsection{Notation and conventions}

With $G$ and $B$ as above, fix a maximal torus $T$ contained in $B$. Let $W=N_{G}(T) / T$ be the Weyl group of $(G, T)$, let $S$ be the set of simple reflections in $W$ determined by the choice of $B$, and let $X(T)$ be the character group of $T$. Then $X(T)$ is a free abelian group and $W$ acts on $X(T)$ as group automorphisms. We use additive notation for $X(T)$ and consider the root system $\Phi$ of $(G, T)$ as a subset of $X(T)$. The 
roots corresponding to the root subgroups in $B$ determine a positive system $\Phi^{+}$, and a base $\Pi$, of $\Phi$. If $s$ is in $S$, then $s=s_{\alpha}$ for a unique $\alpha$ in $\Pi$.

Let $H$ be a complex linear algebraic group. We use the convention that a lowercase fraktur letter denotes the Lie algebra of the group denoted by the same uppercase roman letter, so for example, $\mathfrak{h}=\operatorname{Lie}(H)$. Let $R(H)$ denote the representation ring of $H$ and let $X(H)$ denote the character group of $H$. Define $\bar{H}$ to be the product of $H$ with the one-dimensional complex torus $\mathbb{C}^{*}$, so $\bar{H}=H \times \mathbb{C}^{*}$. Let $v: \bar{H} \rightarrow \mathbb{C}^{*}$ be the character defined by $v(h, z)=z$ for $h \in H$ and $z \in \mathbb{C}^{*}$ and let $A=\mathbb{Z}\left[v, v^{-1}\right]$ be the subring of $R(\bar{H})$ generated by $v$. With this notation, there is a natural isomorphism $R(\bar{H}) \cong A \otimes_{\mathbb{Z}} R(H)$. In particular,

$$
R(\bar{T}) \cong A \otimes_{\mathbb{Z}} R(T) \cong A[X(T)] \quad \text { and } \quad R(\bar{G}) \cong A \otimes_{\mathbb{Z}} R(G) .
$$

When the group $H$ acts on a quasiprojective variety $Y$, let $K^{H}(Y)$ denote the Grothendieck group of the abelian category of $H$-equivariant coherent sheaves on $Y$. The group $K^{H}(Y)$ is naturally an $R(H)$-module.

Suppose $C$ is a closed subgroup of $H$. For a $C$-variety $F$, let $H \times^{C} F$ denote the quotient of $H \times F$ by the $C$-action given by $c \cdot(h, y)=\left(h c^{-1}, c y\right)$ for $c \in C, h \in H$, and $y \in F$. The image of $(h, y)$ in $H \times{ }^{C} F$ is denoted by $h * y$. The group $H$ acts on $H \times{ }^{C} F$ by left multiplication and the projection $f: H \times{ }^{C} F \rightarrow H / C$ given by $h * y \mapsto h C$ is a well-defined $H$-equivariant morphism. Conversely, suppose $Y$ is an $H$-variety and $f_{Y}: Y \rightarrow H / C$ is an $H$-equivariant morphism. Set $F=f_{Y}^{-1}(C)$. Then the map $m: H \times{ }^{C} F \rightarrow Y$ given by $h * y \mapsto h y$ is a well-defined $H$-equivariant isomorphism such that $f=f_{Y} m$. Suppose $\bar{C}$ acts on $F$. Then $\bar{H}$ acts on both $\bar{H} \times{ }^{\bar{C}} F$ and $H \times{ }^{C} F$, and these varieties are canonically isomorphic $\bar{H}$-varieties. It follows from work of Thomason [19, Proposition 6.2] that $K^{\bar{H}}\left(H \times^{C} F\right)$ is naturally isomorphic to $K^{\bar{C}}(F)$, and that if $C_{\mathrm{r}}$ is a reductive subgroup of $C$ such that $C \cong C_{\mathrm{u}} \rtimes C_{\mathrm{r}}$, where $C_{\mathrm{u}}$ is the unipotent radical of $C$, then $K^{\bar{C}}(F)$ is isomorphic to $K^{\bar{C}_{\mathrm{r}}}(F)$ (see [2, §5.2]). Let

$$
\operatorname{res}_{F}: K^{\bar{H}}\left(H \times{ }^{C} F\right) \stackrel{\cong}{\longrightarrow} K^{\bar{C}_{\mathrm{r}}}(F)
$$

denote the composition of these two isomorphisms.

Suppose $Y_{1}$ and $Y_{2}$ are $H$-varieties with $Y_{1} \subseteq Y_{2}$. To simplify the notation, if $Y_{1}$ is closed in $Y_{2}$, then we sometimes denote the direct image map $K^{H}\left(Y_{1}\right) \rightarrow K^{H}\left(Y_{2}\right)$ simply by ()$_{*}$, and if $Y_{1}$ is open in $Y_{2}$, then we sometimes denote the restriction map $K^{H}\left(Y_{2}\right) \rightarrow K^{H}\left(Y_{1}\right)$ by ()$^{*}$.

Unless otherwise indicated, we consider $\mathfrak{g}$ as a $\mathbb{C}^{*}$-module with the action of $\mathbb{C}^{*}$ given by $z \cdot x=z^{-2} x$ for $z$ in $\mathbb{C}^{*}$ and $x$ in $\mathfrak{g}$. Then $\bar{G}$ acts on $\mathfrak{N}$ by $(g, z) \cdot x=z^{-2} g \cdot x$. For a subgroup $P$ of $G, \bar{G}$ acts on $G / P$ by $(g, z) \cdot h P=g h P$. Define

$$
\widetilde{\mathfrak{N}}=\left\{(x, g B) \in \mathfrak{N} \times G / B \mid g^{-1} x \in \mathfrak{b}\right\} .
$$

As above, the Steinberg variety of $G$ is

$$
Z=\left\{(x, g B, h B) \in \mathfrak{N} \times G / B \times G / B \mid g^{-1} x, h^{-1} x \in \mathfrak{b}\right\} \cong \widetilde{\mathfrak{N}} \times_{\mathfrak{N}} \widetilde{\mathfrak{N}}
$$


Then $\bar{G}$ acts on $\widetilde{\mathfrak{N}}$ and $Z$ via the diagonal action, and the projections

$$
p_{Z}: Z \rightarrow \mathfrak{N}, \quad q_{Z}: Z \rightarrow G / B \times G / B, \quad p: \widetilde{\mathfrak{N}} \rightarrow \mathfrak{N}, \quad \text { and } \quad q: \widetilde{\mathfrak{N}} \rightarrow G / B
$$

are all $\bar{G}$-equivariant.

\section{Statement of the MAin theOREM}

\subsection{Generalized Steinberg varieties}

For $I \subseteq S$, let $W_{I}=\langle I\rangle$ be the subgroup of $W$ generated by $I$ and let $P_{I}$ be the parabolic subgroup of $G$ that contains $B$ such that $N_{P_{I}}(T) / T=W_{I}$. Let $U_{I}$ denote the unipotent radical of $P_{I}$ and let $L_{I}$ be the Levi factor of $P_{I}$ that contains $T$. Then $P_{I}=L_{I} U_{I}$ and $\mathfrak{p}_{I}=\mathfrak{l}_{I}+\mathfrak{u}_{I}$ are Levi decompositions of $P_{I}$ and $\mathfrak{p}_{I}$, respectively. Define $\Pi_{I}=\left\{\alpha \in \Pi \mid s_{\alpha} \in I\right\}$ and let $\Phi_{I}$ be the intersection of $\Phi$ with the span of $\Pi_{I}$. Then, with respect to the action of $T, \Phi_{I}$ is the set of roots of $\mathfrak{l}_{I}, \Phi^{+} \cup \Phi_{I}$ is the set of roots of $\mathfrak{p}_{I}$, and $\Phi^{+} \backslash \Phi_{I}$ is the set of roots of $\mathfrak{u}_{I}$. In the special case when $I=\emptyset$, $P_{I}=B$ and we define $U=U_{\emptyset}$.

Each pair of subsets $I, J \subseteq S$ determines a generalized Steinberg variety

$$
X^{I J}=\left\{\left(x, g P_{I}, h P_{J}\right) \in \mathfrak{N} \times G / P_{I} \times G / P_{J} \mid g^{-1} x \in \mathfrak{p}_{I}, h^{-1} x \in \mathfrak{p}_{J}\right\}
$$

(see $[7, \S 2])$. Define

$$
\eta^{I J}: Z \rightarrow X^{I J} \quad \text { by } \quad \eta^{I J}(x, g B, h B)=\left(x, g P_{I}, h P_{J}\right) .
$$

Then $X^{I J}$ is a $\bar{G}$-variety $\left(\bar{G}\right.$ acts diagonally on $X^{I J}$ ) and $\eta^{I J}$ is a surjective, proper, $\bar{G}$-equivariant morphism. Notice that

- if $I=J=\emptyset$, then $X^{I J}=Z$ and $\eta^{I J}$ is the identity, and

- if $I=J=S$, then $X^{I J} \cong \mathfrak{N}$ and $\eta^{I J}$ may be identified with projection $p_{Z}: Z \rightarrow \mathfrak{N}$.

\subsection{Hecke algebras}

The Iwahori-Hecke algebra of $W$ is the $A$-algebra $\mathcal{H}_{S}$ with $A$-basis $T_{w}$, for $w$ in $W$, and multiplication satisfying

$$
\begin{cases}T_{w} T_{w^{\prime}}=T_{w w^{\prime}} & \text { if } \ell\left(w w^{\prime}\right)=\ell(w)+\ell\left(w^{\prime}\right), \text { and } \\ T_{s}^{2}=v^{2} T_{1}+\left(v^{2}-1\right) T_{s} & \text { for } s \text { in } S,\end{cases}
$$

where $\ell$ is the length function on $W$ determined by $S$ and the subscript 1 in $T_{1}$ denotes the identity in $W$ (see [11]).

The extended affine Hecke algebra of $W$ is the $A$-algebra $\mathcal{H}$ with generators $T_{w}, \theta_{\lambda}$, for $w$ in $W$ and $\lambda$ in $X(T)$, and multiplication satisfying

$$
\begin{cases}T_{w} T_{w^{\prime}}=T_{w w^{\prime}} & \text { if } \ell\left(w w^{\prime}\right)=\ell(w)+\ell\left(w^{\prime}\right), \\ T_{s}^{2}=v^{2} T_{1}+\left(v^{2}-1\right) T_{s} & \text { for } s \text { in } S, \\ \theta_{\lambda} \theta_{\mu}=\theta_{\lambda+\mu} & \text { for } \lambda, \mu \text { in } X(T), \\ \theta_{\lambda} T_{s}-T_{s} \theta_{s(\lambda)}=\left(v^{2}-1\right) \frac{\theta_{\lambda}-\theta_{s(\lambda)}}{1-\theta_{-\alpha}} & \text { for } \lambda \text { in } X(T) \text { and } s=s_{\alpha} \text { in } S, \text { and } \\ \theta_{0}=T_{1} & \text { is the identity in } \mathcal{H} .\end{cases}
$$


(See $[15, \S 1]$. Note that for $w$ in $W$, the generator $T_{w}$ in the preceding definition is related to the generator $\tilde{T}_{w}$ in $[15, \S 1]$ by $\left.\tilde{T}_{w}=v^{-\ell(w)} T_{w}.\right)$

We identify the $A$-span, in $\mathcal{H}$, of $\left\{T_{w} \mid w \in W\right\}$ with the Iwahori-Hecke algebra $\mathcal{H}_{S}$, and we identify the $A$-span, in $\mathcal{H}$, of $\left\{\theta_{\lambda} \mid \lambda \in X(T)\right\}$ with the group algebra $A[X(T)]$ of $X(T)$. Then $\mathcal{H}_{S}$ and $A[X(T)]$ are subalgebras of $\mathcal{H}$ that contain the identity. The center of $\mathcal{H}$ is $A[X(T)]^{W}$ (see [14]). We identify $R(\bar{G})$ with $A[X(T)]^{W}$, and hence with the center of $\mathcal{H}$, via the isomorphism $R(\bar{G}) \cong A[X(T)]^{W}$ given by associating with a representation of $\bar{G}$ its character in $A[X(T)]$. The map $A[X(T)] \otimes_{A} \mathcal{H}_{S} \rightarrow \mathcal{H}$ given by multiplication, $\theta_{\lambda} \otimes T_{w} \mapsto \theta_{\lambda} T_{w}$, is an $A$-module isomorphism. We call $\left\{\theta_{\lambda} T_{w} \mid \lambda \in X(T), w \in W\right\}$ the Bernstein basis of $\mathcal{H}$ because it arises from the Bernstein presentation (2.2).

For $\lambda$ in $X(T)$, let $t_{\lambda}$ denote translation by $\lambda$ in $X(T)$. Then $\left\{t_{\lambda} \mid \lambda \in X(T)\right\}$ is a subgroup of $\operatorname{Aut}(X(T))$ isomorphic to $X(T)$. Recall that $W$ acts faithfully on $X(T)$. Define $W_{\text {ex }}$, the extended affine Weyl group of $\Phi$, to be the subgroup of $\operatorname{Aut}(X(T))$ generated by the image of $W$ and $\left\{t_{\lambda} \mid \lambda \in X(T)\right\}$. Then $W_{\text {ex }}$ is isomorphic to the semi-direct product $X(T) \rtimes W$. We frequently identify $W$ with its image in $\operatorname{Aut}(X(T))$ and consider $W$ as a subgroup of $W_{\mathrm{ex}}$.

The affine Weyl group of $\Phi, W_{\text {af }}$, is the subgroup of $\operatorname{Aut}(X(T))$ generated by the image of $W$ and $\left\{t_{\alpha} \mid \alpha \in \Phi\right\}$. Then $W_{\text {af }}$ is a normal subgroup of $W_{\text {ex }}$ and there is a finite abelian subgroup $\Gamma$ of $W_{\text {ex }}$ such that $W_{\text {ex }}=W_{\text {af }} \Gamma$ and $W_{\text {af }} \cap \Gamma=1$. The group $W_{\text {af }}$ is a Coxeter group with a Coxeter generating set $S_{\text {af }}$ that contains $S$, and $\Gamma$ acts on $W_{\text {af }}$ as Coxeter group automorphisms preserving $S_{\text {af }}$. Extend the length function $\ell$ and Bruhat order $\leq$ on $W_{\text {af }}$ to $W_{\text {ex }}$ by defining

$$
\ell(y \gamma)=\ell(y) \quad \text { and } \quad y \gamma \leq y^{\prime} \gamma^{\prime} \text { if and only if } \gamma=\gamma^{\prime} \text { and } y \leq y^{\prime}
$$

for $y, y^{\prime}$ in $W_{\text {af }}$ and $\gamma, \gamma^{\prime}$ in $\Gamma$ (see $\left.[14, \S 2]\right)$.

The algebra $\mathcal{H}$ has a standard basis, $\left\{T_{w} \mid w \in W_{\text {ex }}\right\}$, such that the relations (2.1) hold (see $[15, \S 1]$ ). The "bar" involution of $\mathcal{H},-: \mathcal{H} \rightarrow \mathcal{H}$, is the ring automorphism of $\mathcal{H}$ defined by $\bar{v}=v^{-1}$ and $\overline{T_{x}}=T_{x^{-1}}^{-1}$ for $x$ in $W_{\text {ex }}$. As observed by Lusztig [14], the argument in the proof of [11, Theorem 1.1] can be applied to show that for $x$ in $W_{\text {ex }}$, there are unique elements $C_{x}$ and $C_{x}^{\prime}$ in $\mathcal{H}$ such that

$$
\left\{\begin{array}{l}
\overline{C_{x}}=C_{x} \\
C_{x}=v_{x}^{-1} T_{x}+\sum_{y<x} \epsilon_{y} \epsilon_{x} v_{x} v_{y}^{-2} \bar{P}_{y, x} T_{y}
\end{array}\right.
$$

and

$$
\left\{\begin{array}{l}
\overline{C_{x}^{\prime}}=C_{x}^{\prime} \\
C_{x}^{\prime}=v_{x}^{-1} T_{x}+\sum_{y<x} v_{x}^{-1} P_{y, x} T_{y}
\end{array}\right.
$$

where

$$
\epsilon_{x}=(-1)^{\ell(x)} \text { and } \quad v_{x}=v^{\ell(x)}
$$

for $x$ in $W_{\mathrm{ex}}$, and $P_{y, x}$ is a polynomial in $v$ of degree at most $\ell(x)-\ell(y)-1$. (See also $[15, \S 1.7,1.8]$.) We call $\left\{C_{x} \mid x \in W_{\text {ex }}\right\}$ and $\left\{C_{x}^{\prime} \mid x \in W_{\text {ex }}\right\}$ Kazhdan-Lusztig 
bases of $\mathcal{H}$. A fundamental property of the Kazhdan-Lusztig bases is that if $x$ is in $W_{\text {ex }}$ and $s, t$ are in $S$ with $\ell(t x)<\ell(x)$ and $\ell(x s)<\ell(x)$, then

$$
T_{t} C_{x}=C_{x} T_{s}=-C_{x} \quad \text { and } \quad T_{t} C_{x}^{\prime}=C_{x}^{\prime} T_{s}=v^{2} C_{x}^{\prime}
$$

For a subset $I$ of $S$ let $w_{I}$ be the longest element in $W_{I}$. Then

$$
C_{w_{I}}=(-v)^{\ell\left(w_{I}\right)} \sum_{y \in W_{I}} \epsilon_{y} v_{y}^{-2} T_{y} \quad \text { and } \quad T_{s} C_{w_{I}}=C_{w_{I}} T_{s}=-C_{w_{I}}
$$

for $s$ in $I$. For subsets $I$ and $J$ of $S$ we have the Kazhdan-Lusztig basis elements $C_{w_{I}}$ and $C_{w_{J}}$ of $\mathcal{H}$. Define

$$
\mathcal{H}^{I J}=C_{w_{I}} \mathcal{H} C_{w_{J}} \quad \text { and } \quad \chi^{I J}: \mathcal{H} \rightarrow \mathcal{H}^{I J} \text { by } \chi^{I J}(h)=C_{w_{I}} h C_{w_{J}} .
$$

Obviously, $\mathcal{H}^{I J}$ is an $R(\bar{G})$-submodule of $\mathcal{H}$ and $\chi^{I J}$ is a surjective $R(\bar{G})$-module homomorphism.

\subsection{The isomorphism $\mathcal{H}^{I J} \cong K^{\bar{G}}\left(X^{I J}\right)$}

The group $\bar{G}$ acts on the Steinberg variety $Z$ and so $K^{\bar{G}}(Z)$ is naturally an $R(\bar{G})$ module, and hence an $A$-module. Chriss and Ginzburg [2, $\$ 7.2]$ and Lusztig [15] have shown that $K^{\bar{G}}(Z)$ has an $A$-algebra structure such that $\mathcal{H} \cong K^{\bar{G}}(Z)$. Let $\varphi: \mathcal{H} \rightarrow K^{\bar{G}}(Z)$ be the $A$-algebra isomorphism constructed by Lusztig [15, Theorem 8.6]. The main result in this paper is the following theorem.

Theorem 2.1. For each pair of subsets $(I, J)$ of $S$ there is a unique $R(\bar{G})$-module isomorphism

$$
\psi^{I J}: \mathcal{H}^{I J} \stackrel{\cong}{\longrightarrow} K^{\bar{G}}\left(X^{I J}\right)
$$

such that the diagram

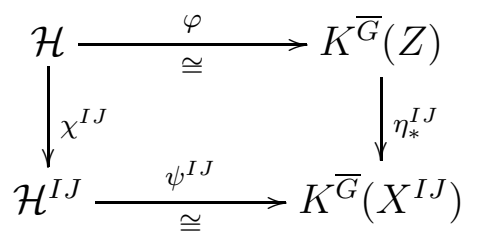

commutes.

In $\S 4$ we use the isomorphism $\psi^{I J}$ to define a standard basis, a "bar" involution, and a Kazhdan-Lusztig basis for $K^{\bar{G}}\left(X^{I J}\right)$. As we explain next, the proof of Theorem 2.1 gives significantly more information about the isomorphism $\psi^{I J}$ than is encoded in (2.6). This leads to a graded refinement of the theorem that is given in Corollary 3.10.

The preimages of the $G$-orbits on $G / P_{I} \times G / P_{J}$ under the projection on the second and third factors, say $\left\{X_{z}^{I J}\right\}$, form a partition of $X^{I J}$ into locally closed, equidimensional subvarieties indexed by $\left\{W_{I} z W_{J}\right\}$, the set of $\left(W_{I}, W_{J}\right)$-double cosets in $W$. The closures of these subvarieties are the irreducible components of $X^{I J}$ (see [7, §3]). Thus, the fundamental classes of the closures of the subvarieties $X_{z}^{I J}$ form a basis of the top Borel-Moore homology of $X^{I J}$. As explained in detail below, the contribution 
of each subvariety $X_{z}^{I J}$ to $K^{\bar{G}}\left(X^{I J}\right)$ is not just a single homology class, rather it is the full equivariant $K$-group of isomorphism classes of $\bar{L}_{I} \cap{ }^{z} \bar{L}_{J}$-equivariant coherent sheaves on the nilpotent cone of $\mathfrak{l}_{I} \cap z \mathfrak{l}_{J}$. A basis of this $K$-group is indexed by the set of $\left(W_{I}, W_{J}\right)$-double cosets in $W_{I} \backslash W_{\text {ex }} / W_{J}$ that project to the double coset $W_{I} z W_{J}$ in $W$. Taking the union over $z$ (in a suitable sense) gives rise to a basis of $K^{\bar{G}}\left(X^{I J}\right)$ indexed by the $\left(W_{I}, W_{J}\right)$-double cosets in $W_{\text {ex }}$.

In case $I=J=\emptyset, X^{\emptyset \emptyset}=Z$, and $\left\{W_{\emptyset} z W_{\emptyset}\right\}=W$. For $w$ in $W, X_{w}^{\emptyset \emptyset}=Z_{w}$ is the conormal bundle to the $G$-orbit in $G / B \times G / B$ corresponding to $w, L_{\emptyset} \cap{ }^{w} L_{\emptyset}=T$, the cone of nilpotent elements in $\mathfrak{l}_{\emptyset} \cap w \mathfrak{l}_{\emptyset}$ is $\{0\}$, and $K^{\bar{T}}(\{0\}) \cong A[X(T)]$. Obviously, $\left\{t_{\lambda} w \mid \lambda \in X(T)\right\}$ parametrizes the set of $\left(W_{\emptyset}, W_{\emptyset}\right)$-double cosets in $W_{\text {ex }}$ that project to $\{w\}$. It follows from Theorem 3.4 that $\left\{t_{\lambda} w \mid \lambda \in X(T)\right\}$ parametrizes an $A$-basis of $K^{\bar{G}}\left(Z_{w}\right)$ and it follows from $[15, \S 8.6]$ that $\left\{t_{\lambda} w \mid w \in W, \lambda \in X(T)\right\}$ parametrizes an $A$-basis of $K^{\bar{G}}(Z)$.

At the other extreme, when $I=J=S$, there is a single $(W, W)$-double coset in $W$ (with representative 1 ) and $\left\{W_{S} z W_{S}\right\}=\{W\}$. In this case, $X_{1}^{S S}=X^{S S \cong \mathfrak{N}}$, $L_{S} \cap{ }^{1} L_{S}=G$, and the cone of nilpotent elements in $\mathfrak{l}_{S} \cap{ }^{1} \mathfrak{l}_{S}$ is $\mathfrak{N}$. The $\left(W_{S}, W_{S}\right)$ double cosets in $W_{\text {ex }}$ that project to $W$ are simply the $(W, W)$-double cosets in $W_{\text {ex }}$. These are parametrized by $\left\{t_{\lambda} \mid \lambda \in X(T)^{+}\right\}$, where $X(T)^{+}$is the set of dominant weights relative to the choice of $B$. For $\lambda$ in $X(T)$ let $\mathcal{L}_{\lambda}$ be the $G$-equivariant line bundle on $G / B$ such that $T$ acts on the fibre over $B$ with character $-\lambda$. We consider $\mathcal{L}_{\lambda}$ as a $\bar{G}$-equivariant line bundle on $G / B$ via the natural projection $\bar{G} \rightarrow G$. It follows from a result of Broer [1] that if $\lambda$ is dominant, then $p_{*} q^{*}\left(\left[\mathcal{L}_{\lambda}\right]\right)=\left[R^{0} p_{*} q^{*} \mathcal{L}_{\lambda}\right]$ in $K^{\bar{G}}(\mathfrak{N})$. Ostrik $[17, \S 2.2]$ has proved the following key lemma he attributes to R. Bezrukavnikov.

Lemma 2.2. The set $\left\{p_{*} q^{*}\left(\left[\mathcal{L}_{\lambda}\right]\right) \mid \lambda \in X(T)^{+}\right\}$is an $A$-basis of $K^{\bar{G}}(\mathfrak{N})$.

In [17] Ostrik assumes that the group $G$ is simple. The extension to reductive groups is straightforward. It follows from the lemma that $\left\{t_{\lambda} \mid \lambda \in X(T)^{+}\right\}$parametrizes an $A$-basis of $K^{\bar{G}}\left(X_{1}^{S S}\right)=K^{\bar{G}}(\mathfrak{N})$.

For arbitrary $I, J \subseteq S$, the results we prove below are an amalgam of the two extreme cases. Let $W^{I J}$ denote the set of minimal length $\left(W_{I}, W_{J}\right)$-double coset representatives in $W$. For $z$ in $W^{I J}$, set

$$
L_{z}=L_{I} \cap^{z} L_{J}
$$

and let $X(T)_{z}^{+}$be the set of weights in $X(T)$ that are dominant for $L_{z}$. Then $L_{z}$ is a reductive group (see $[5, \S 69 \mathrm{~B}]$ ). We show in Theorem 3.4 that there is an isomorphism $K^{\bar{G}}\left(X_{z}^{I J}\right) \cong K^{\bar{L}_{z}}\left(\mathfrak{N}_{z}\right)$, where $\mathfrak{N}_{z}$ is the nilpotent cone in $\mathfrak{l}_{z}$ and we show in Lemma 3.6 that $\left\{t_{\lambda} z \mid \lambda \in X(T)_{z}^{+}\right\}$parametrizes the set of $\left(W_{I}, W_{J}\right)$-double cosets in $W_{\mathrm{ex}}$ that project to $W_{I} z W_{J}$. It follows from Theorem 3.8 that $\left\{t_{\lambda} z \mid \lambda \in g X(T)_{z}^{+}\right\}$ parametrizes an $A$-basis of $K^{\bar{G}}\left(X_{z}^{I J}\right)$ and it follows from Corollary 3.9 that $\left\{t_{\lambda} z \mid z \in\right.$ $\left.W^{I J}, \lambda \in X(T)_{z}^{+}\right\}$parametrizes an $A$-basis of $K^{\bar{G}}\left(X^{I J}\right)$.

In summary, there is a filtration of $K^{\bar{G}}\left(X^{I J}\right)$ such that the direct summands of the associated graded $A$-module are naturally indexed by $\left(W_{I}, W_{J}\right)$-double cosets in $W$. 
In addition, the summand indexed by $z$ in $W^{I J}$ is isomorphic to $K^{\bar{L}_{z}}\left(\mathfrak{N}_{z}\right)$ and has a basis consisting of isomorphism classes of equivariant coherent sheaves canonically indexed by the $\left(W_{I}, W_{J}\right)$-double cosets in $W_{\text {ex }}$ that project to $W_{I} z W_{J}$.

\section{The Proof of TheOREM 2.1}

In this section we prove Theorem 2.1. The proof proceeds in three steps. The first step is show that $K^{\bar{G}}\left(X^{I J}\right)$ is a free $A$-module, the second step is to show that the composition $\eta_{*}^{I J} \varphi$ factors through $\chi^{I J}$, and the third step is to show that the resulting map $\psi^{I J}$ is an isomorphism. In the course of the argument we construct explicit $A$-bases of of $\mathcal{H}^{I J}$ and $K^{\bar{G}}\left(X^{I J}\right)$ that correspond under $\psi^{I J}$.

To show that $K^{\bar{G}}\left(X^{I J}\right)$ is a free $A$-module, we use the filtration on $K^{\bar{G}}\left(X^{I J}\right)$ determined by the $G$-orbits on $G / P_{I} \times G / P_{J}$. Recall that the rule $z \mapsto G \cdot\left(P_{I}, z P_{J}\right)$ defines a bijection between $W^{I J}$ and the set of $G$-orbits in $G / P_{I} \times G / P_{J}$. Let $q_{I J}: X^{I J} \rightarrow G / P_{I} \times G / P_{J}$ be the projection on the second and third factors, and for $z$ in $W^{I J}$ define $X_{z}^{I J}$ to be the preimage in $X^{I J}$ of the orbit $G \cdot\left(P_{I}, z P_{J}\right)$. Then

$$
X_{z}^{I J}=\left\{\left(x, g P_{I}, g z P_{J}\right) \in \mathfrak{N} \times G / P_{I} \times G / P_{J} \mid g^{-1} x \in \mathfrak{N} \cap \mathfrak{p}_{I} \cap z \mathfrak{p}_{J}\right\} .
$$

Choose a linear order on $W^{I J}$, say $W^{I J}=\left\{z_{i}|1 \leq i \leq| W^{I J} \mid\right\}$, that extends the Bruhat order and define

$$
X_{\leq i}^{I J}=\coprod_{j \leq i} X_{z_{j}}^{I J}
$$

Then $X_{\leq i}^{I J}=X_{\leq i-1}^{I J} \amalg X_{z_{i}}^{I J}$, where $X_{\leq i-1}^{I J}$ is closed in $X_{\leq i}^{I J}$ and $X_{z_{i}}^{I J}$ is open in $X_{\leq i}^{I J}$. It is shown in $\oint 8.2$ that for $i \geq 1$ the sequence

$$
0 \longrightarrow K^{\bar{G}}\left(X_{\leq i-1}^{I J}\right) \stackrel{()_{*}}{\longrightarrow} K^{\bar{G}}\left(X_{\leq i}^{I J}\right) \stackrel{()^{*}}{\longrightarrow} K^{\bar{G}}\left(X_{z_{i}}^{I J}\right) \longrightarrow 0
$$

of $R(\bar{G})$-modules is exact. It follows that the embedding $X_{\leq i}^{I J} \hookrightarrow X^{I J}$ induces an injection $K^{\bar{G}}\left(X_{\leq i}^{I J}\right) \hookrightarrow K^{\bar{G}}\left(X^{I J}\right)$ in equivariant $K$-theory.

Suppose $z$ is in $W^{I J}$ and recall that $L_{z}=L_{I} \cap{ }^{z} L_{J}$ and that $\mathfrak{N}_{z}=\mathfrak{N} \cap \mathfrak{l}_{z}$ is the cone of nilpotent elements in $\mathfrak{l}_{z}$. It is shown in $\S 8.1$, in the course of the proof of Theorem 3.4, that $K^{\bar{G}}\left(X_{z}^{I J}\right) \cong K^{\bar{L}_{z}}\left(\mathfrak{N}_{z}\right)$. Thus, the next proposition follows from the exact sequence (3.1) by induction and Lemma 2.2.

Proposition 3.1. The equivariant $K$-group $K^{\bar{G}}\left(X^{I J}\right)$ is a free A-module.

Next, to show that $\eta_{*}^{I J} \varphi$ factors through $\chi^{I J}$, it is enough to show that for each generator $s$ in $J$, and each generator $t$ in $I$,

$$
\eta_{*}^{I J} \varphi\left(h T_{s}\right)=-\eta_{*}^{I J} \varphi(h)=\eta_{*}^{I J} \varphi\left(T_{t} h\right)
$$

for all $h$ in $\mathcal{H}$. Indeed, if this condition holds, then for all $h$ in $\mathcal{H}, x$ in $W_{I}$, and $y$ in $W_{J}, \eta_{*}^{I J} \varphi\left(T_{x} h T_{y}\right)=\epsilon_{x} \epsilon_{y} \eta_{*}^{I J} \varphi(h)$, and so $\eta_{*}^{I J} \varphi\left(C_{w_{I}} h C_{w_{J}}\right)=r_{I J} \eta_{*}^{I J} \varphi(h)$, where $r_{I J}$ is a non-zero element of $A$ that depends only on $I$ and $J$. Then $\psi^{I J}: \mathcal{H}^{I J} \rightarrow K^{\bar{G}}\left(X^{I J}\right)$ by $\psi^{I J}\left(C_{w_{I}} h C_{w_{J}}\right)=\eta_{*}^{I J} \varphi(h)$ is well defined because if $C_{w_{I}} h C_{w_{J}}=0$, then $r_{I J} \eta_{*}^{I J} \varphi(h)=0$ and so $\eta_{*}^{I J} \varphi(h)=0$. 
Let $\pi_{I}: X^{\emptyset J} \rightarrow X^{I J}$ by $\pi_{I}\left(x, g B, h P_{J}\right)=\left(x, g P_{I}, h P_{J}\right)$ and let $\pi_{J}: X^{I \emptyset} \rightarrow X^{I J}$ by $\pi_{J}\left(x, g P_{I}, h B\right)=\left(x, g P_{I}, h P_{J}\right)$. Then the diagram

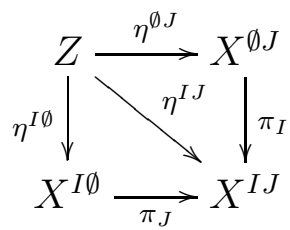

commutes and so (3.2) follows from the equalities

$$
\eta_{*}^{\emptyset J} \varphi\left(h T_{s}\right)=-\eta_{*}^{\emptyset J} \varphi(h) \text { and } \eta_{*}^{I \emptyset} \varphi\left(T_{t} h\right)=-\eta_{*}^{I \emptyset} \varphi(h),
$$

for $s \in J$ and $t \in I$, by applying $\left(\pi_{I}\right)_{*}$ and $\left(\pi_{J}\right)_{*}$, respectively. Thus, by symmetry, it is enough to show that $\eta_{*}^{\emptyset J} \varphi$ factors through the projection $\mathcal{H} \rightarrow \mathcal{H} C_{w_{J}}$ given by right multiplication by $C_{w_{J}}$ :

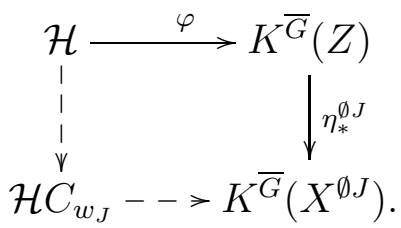

The intersection/Tor-product construction described by Lusztig in [15, §6.4] can be used to define a $K^{\bar{G}}(Z)$-module structure on $K^{\bar{G}}\left(X^{\emptyset J}\right)$, say

$$
\star_{J}: K^{\bar{G}}(Z) \times K^{\bar{G}}\left(X^{\emptyset J}\right) \rightarrow K^{\bar{G}}\left(X^{\emptyset J}\right),
$$

such that the map $\eta_{*}^{\emptyset J}: K^{\bar{G}}(Z) \rightarrow K^{\bar{G}}\left(X^{\emptyset J}\right)$ is $K^{\bar{G}}(Z)$-linear (see Proposition 5.2). Thus, for all $h$ in $\mathcal{H}$ and all $s$ in $S$,

$$
\eta_{*}^{\emptyset J} \varphi\left(h T_{s}\right)=\eta_{*}^{\emptyset J}\left(\varphi(h) \star \varphi\left(T_{s}\right)\right)=\varphi(h) \star_{J} \eta_{*}^{\emptyset J}\left(\varphi\left(T_{s}\right)\right),
$$

where $\star$ is the convolution product in $K^{\bar{G}}(Z)$. Hence, it is enough to show that $\eta_{*}^{\emptyset J}\left(\varphi\left(T_{s}\right)\right)=-\eta_{*}^{\emptyset J}(\varphi(1))$ for all $s$ in $J$, or equivalently that $\eta_{*}^{\emptyset J}\left(\varphi\left(T_{s}+1\right)\right)=0$. For a simple reflection $s$ in $W$, let $\mathbf{a}_{s}$ in $K^{\bar{G}}(Z)$ be defined as in $[15, \S 7.20]$ (see $\S 6$ ). By [15, $\S 7.25], \varphi\left(T_{s}+1\right)=-v \mathbf{a}_{s}$. Therefore, the existence of the map $\psi^{I J}$ in Theorem 2.1 follows from the next theorem.

Theorem 3.2. Suppose $s$ is in $J$. Then $\eta_{*}^{\emptyset J}\left(\mathbf{a}_{s}\right)=0$ in $K^{\bar{G}}\left(X^{\emptyset J}\right)$.

This theorem is proved in $\S 6$.

Finally, to show that $\psi^{I J}$ is an isomorphism, we use an $A$-basis of $\mathcal{H}^{I J}$ and the partition $X^{I J}=\coprod_{z \in W^{I J}} X_{z}^{I J}$ to define compatible filtrations on $\mathcal{H}^{I J}$ and $K^{\bar{G}}\left(X^{I J}\right)$. We also need the analogous constructions for $\mathcal{H}$ and $K^{\bar{G}}(Z)$.

Recall that $q_{Z}: Z \rightarrow G / B \times G / B$ is the projection on the second and third factors. For $w$ in $W$ define $Z_{w}$ to be the preimage in $Z$ of the orbit $G \cdot(B, w B)$. Then

$$
Z_{w}=\left\{(x, g B, g w B) \in \mathfrak{N} \times G / B \times G / B \mid g^{-1} x \in \mathfrak{u} \cap w \mathfrak{u}\right\} .
$$


Define

$$
Z_{\leq w}=\coprod_{y \leq w} Z_{y} \quad \text { and } \quad Z_{<w}=Z_{\leq w} \backslash Z_{w}=\coprod_{y<w} Z_{y}
$$

where $\leq$ is the Bruhat order on $W$. Similarly, for $z$ in $W^{I J}$ define

$$
X_{\leq z}^{I J}=\coprod_{y \leq z} X_{y}^{I J} \quad \text { and } \quad X_{<z}^{I J}=X_{\leq z}^{I J} \backslash X_{z}^{I J}=\coprod_{y<z} X_{y}^{I J},
$$

where the unions are over $y$ in $W^{I J}$.

Suppose $z$ is in $W^{I J}$ and let $\eta^{z}, \eta^{\leq z}$, and $\eta^{<z}$ be the restrictions of $\eta^{I J}$ to $Z_{z}, Z_{\leq z}$, and $Z_{<z}$, respectively. It is shown in [7, §3] that if $w_{1}$ is in $W_{I}$ and $w_{2}$ is in $W_{J}$, then $\eta^{I J}\left(Z_{w_{1} z w_{2}}\right) \subseteq X_{z}^{I J}$, and that $\eta^{I J}\left(Z_{w_{1} z w_{2}}\right)=X_{z}^{I J}$ if and only if $w_{1} z w_{2}=z$. It is shown in [6, Lemma 2.2] that if $z$ and $z^{\prime}$ are in $W^{I J}$, then $z \leq z^{\prime}$ if and only if there are elements $w$ in $W_{I} z W_{J}$ and $w^{\prime}$ in $W_{I} z^{\prime} W_{J}$ such that $w \leq w^{\prime}$. Therefore, $\eta^{I J}\left(Z_{\leq z}\right) \subseteq X_{\leq z}^{I J}$, and letting $r_{z}$ and $r_{z}^{I J}$ denote the inclusions $Z_{z} \rightarrow Z_{\leq z}$ and $X_{z}^{I J} \rightarrow$ $X_{\leq z}^{I J}$, respectively, the square

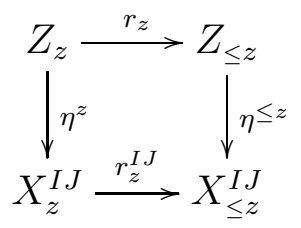

is cartesian.

Lemma 3.3. The map $\eta^{z}: Z_{z} \rightarrow X_{z}^{I J}$ is a proper morphism.

Proof. Define $Z_{W_{I} z W_{J}}=\coprod_{w \in W_{I} z W_{J}} Z_{w}$. Then $Z_{W_{I} z W_{J}}=\left(\eta^{I J}\right)^{-1}\left(X_{z}^{I J}\right)$ and so by base change, the restriction of $\eta^{I J}$ to $Z_{W_{I} z W_{J}}$ is proper. Now $G \cdot(B, z B)$ is closed in $\coprod_{w \in W_{I} z W_{J}} G \cdot(B, w B)$ and hence $Z_{z}$ is closed in $Z_{W_{I} z W_{J}}$. It follows that $\eta^{z}$ is proper.

Since $\eta^{I J}\left(Z_{\leq z}\right) \subseteq X_{\leq z}^{I J}$ for all $z$ in $W^{I J}$ and (3.3) is cartesian, the proper morphisms $\eta^{I J}$ and $\eta^{z}$ induce a map of short exact sequences such that the following diagram commutes:

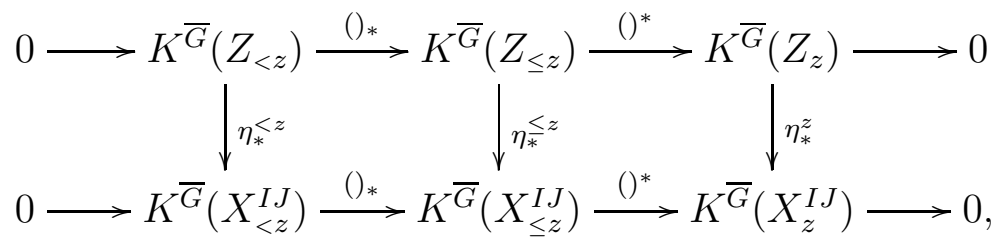

where $Z_{<1}=X_{<1}^{I J}=\emptyset$.

Suppose $z$ is in $W^{I J}$. Then $L_{z}=L_{I} \cap{ }^{z} L_{J}=L_{I \cap \cap_{J}}$ is a Levi subgroup of $P_{I} \cap{ }^{z} P_{J}$ (see $[5, \S 69 \mathrm{~B}])$. Set

$$
B_{z}=L_{z} \cap B \quad \text { and } \quad \widetilde{\mathfrak{N}}_{z}=\left\{\left(x, h B_{z}\right) \in \mathfrak{N}_{z} \times L_{z} / B_{z} \mid h^{-1} x \in \mathfrak{b}_{z}\right\}
$$

and let

$$
p_{z}: \tilde{\mathfrak{N}}_{z} \rightarrow \mathfrak{N}_{z} \quad \text { and } \quad q_{z}: \tilde{\mathfrak{N}}_{z} \rightarrow L_{z} / B_{z}
$$


be the projections. Then $B_{z}$ is a Borel subgroup of $L_{z}$ and $p_{z}$ is the Springer resolution of $\mathfrak{N}_{z}$.

Theorem 3.4. Suppose $z$ is in $W^{I J}$. Then there is a commutative diagram of $R(\bar{G})$ modules

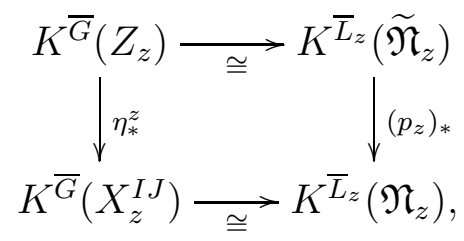

where the horizontal maps are isomorphisms and the vertical maps are surjections.

This theorem is proved in $\S 8.1$.

Corollary 3.5. The map $\eta_{*}^{\leq z}: K^{\bar{G}}\left(Z_{\leq z}\right) \rightarrow K^{\bar{G}}\left(X_{\leq z}^{I J}\right)$ is surjective for all $z$ in $W^{I J}$. Therefore, the maps $\eta_{*}^{I J}: K^{\bar{G}}(Z) \rightarrow K^{\bar{G}}\left(X^{I J}\right)$ and $\psi^{I J}: \mathcal{H}^{I J} \rightarrow K^{\bar{G}}\left(X^{I J}\right)$ are both surjective.

Proof. We show that $\eta_{*}^{\leq z}$ is surjective using induction on $z$ in the Bruhat order on $W^{I J}$. It is shown in $[12, \S 3.17]$ that the image of $K^{\bar{G}}\left(Z_{<z}\right)$ in $K^{\bar{G}}(Z)$ coincides with the sum of the images of the spaces $K^{\bar{G}}\left(Z_{\leq y}\right)$ for $y<w$. A similar argument, using Lemma 8.2, shows that the image of $K^{\bar{G}}\left(X_{<z}^{I J}\right)$ in $K^{\bar{G}}\left(X^{I J}\right)$ coincides with the sum of the images of the spaces $K^{\bar{G}}\left(X_{\leq y}^{I J}\right)$ for $y$ in $W^{I J}$ with $y<z$. Thus, the natural maps

$$
\bigoplus_{y<z} K^{\bar{G}}\left(Z_{\leq y}\right) \stackrel{f_{1}}{\longrightarrow} K^{\bar{G}}\left(Z_{<z}\right) \text { and } \bigoplus_{\substack{y \in W^{I J} \\ y<z}} K^{\bar{G}}\left(X_{\leq y}^{I J}\right) \stackrel{f_{2}}{\longrightarrow} K^{\bar{G}}\left(X_{<z}^{I J}\right)
$$

are surjective. The composition $\bigoplus_{y<z} K^{\bar{G}}\left(Z_{\leq y}\right) \stackrel{f_{1}}{\longrightarrow} K^{\bar{G}}\left(Z_{<z}\right) \stackrel{\eta_{*}^{<z}}{\longrightarrow} K^{\bar{G}}\left(X_{<z}^{I J}\right)$ is equal the composition $\bigoplus_{y<z} K^{\bar{G}}\left(Z_{\leq y}\right) \rightarrow \bigoplus_{\substack{y \in W^{I J} \\ y<z}} K^{\bar{G}}\left(X_{\leq y}^{I J}\right) \stackrel{f_{2}}{\longrightarrow} K^{\bar{G}}\left(X_{<z}^{I J}\right)$. By induction, and the fact that the map $f_{2}$ is surjective, this last composition is surjective. It follows that $\eta_{*}^{<z}$ is surjective. Now the fact that $\eta_{*}^{\leq z}$ is surjective follows from diagram (3.4) by the Five Lemma and Theorem 3.4.

Let $z_{0}$ be the element in $W^{I J}$ with maximal length. Then $X_{\leq z_{0}}^{I J}=X^{I J}$ and so taking $z=z_{0}$ we see that the map $K^{\bar{G}}\left(Z_{\leq z_{0}}\right) \rightarrow K^{\bar{G}}\left(X^{I J}\right)$ induced by $\eta^{I J}$ is a surjection. This map is $\eta_{*}^{I J} \circ\left(j_{z_{0}}\right)_{*}$, where $j_{z_{0}}: Z_{\leq z_{0}} \rightarrow Z$ is the inclusion. Therefore, $\eta_{*}^{I J}$ is surjective and so $\psi^{I J}$ is surjective.

To define filtrations on $\mathcal{H}$ and $\mathcal{H}^{I J}$ compatible with diagram (3.4) we need to study $\left(W_{I}, W_{J}\right)$-double cosets in $W_{\mathrm{ex}}$. For $I \subseteq S$ define

$$
X(T)_{I}^{+}=\left\{\lambda \in X(T) \mid\langle\lambda, \check{\alpha}\rangle \geq 0 \forall \alpha \in \Pi_{I}\right\},
$$

where $\langle\cdot, \cdot\rangle$ is the usual pairing between $X(T)$ and $\operatorname{Hom}\left(\mathbb{C}^{*}, T\right)$, the group of cocharacters of $T$, and $\{\check{\alpha} \mid \alpha \in \Phi\}$ is the dual root system in $\operatorname{Hom}\left(\mathbb{C}^{*}, T\right)$. Then $X(T)_{\emptyset}^{+}=X(T)$ and $X(T)_{S}^{+}=X(T)^{+}$, the set of dominant weights. Because $X(T)^{+}$is 
a fundamental domain for the action of $W$ on $X(T)$, it follows that $\left\{t_{\lambda} \mid \lambda \in X(T)^{+}\right\}$ is a set of $(W, W)$-double coset representatives in $W_{\mathrm{ex}}$. The next lemma is the analog of this fact for $\left(W_{I}, W_{J}\right)$-double cosets.

Lemma 3.6. Every $\left(W_{I}, W_{J}\right)$-double coset in $W_{\mathrm{ex}}$ contains a unique representative of the form $t_{\lambda} z$, where $z$ is in $W^{I J}$ and $\lambda$ is in $X(T)_{I \cap z J}^{+}$.

Proof. If $\lambda$ is in $X(T), w$ is in $W, w_{1}$ is in $W_{I}$, and $w_{2}$ is in $W_{J}$, then $w_{1} t_{\lambda} w w_{2}=$ $t_{w_{1}(\lambda)} w_{1} w w_{2}$. Thus, every $\left(W_{I}, W_{J}\right)$-double coset has a representative of the form $t_{\lambda} z$ where $z$ is in $W^{I J}$. Suppose that $z$ is in $W^{I J}$ and $\lambda$ and $\mu$ are in $X(T)$. Then $t_{\mu} z=w_{1} t_{\lambda} z w_{2}$ for some $w_{1}$ in $W_{I}$ and $w_{2}$ in $W_{J}$ if and only if $\mu=w_{1}(\lambda)$ and $z=w_{1} z w_{2}$. Therefore, $t_{\lambda} z$ and $t_{\mu} z$ are in the same $\left(W_{I}, W_{J}\right)$-double coset if and only if $\mu=w_{1}(\lambda)$ for some $w_{1}$ in $W_{I} \cap{ }^{z} W_{J}$. Because $W_{I} \cap{ }^{z} W_{J}=W_{I \cap z J}$ (see $[5, \S 64 \mathrm{C}]$ ), we see that $t_{\lambda} z$ and $t_{\mu} z$ are in the same $\left(W_{I}, W_{J}\right)$-double coset if and only if $\mu=w_{1}(\lambda)$ for some $w_{1}$ in $W_{I \cap^{z} J}$. Finally, $X(T)_{I \cap^{z} J}^{+}$is a fundamental domain for the action of $W_{I \cap^{z} J}$ on $X(T)$ and so every $\left(W_{I}, W_{J}\right)$-double coset in $W_{\text {ex }}$ contains a unique representative of the form $t_{\lambda} z$, where $z$ is in $W^{I J}$ and $\lambda$ is in $X(T)_{I \cap \cap^{z}}^{+}$.

For $z$ in $W^{I J}$, set

$$
X(T)_{z}^{+}=X(T)_{I \cap z J}^{+}
$$

Then $X(T)_{z}^{+}$is the set of weights that are dominant for $L_{z}$ with respect to the Borel subgroup $B_{z}$.

Let $\epsilon_{I}=\sum_{w \in W_{I}} \epsilon_{w} w$ for $I \subseteq S$. Then Lemma 3.6 shows that $\left\{\epsilon_{I} t_{\lambda} z \epsilon_{J} \mid z \in\right.$ $\left.W^{I J}, \lambda \in X(T)_{z}^{+}\right\}$is a $\mathbb{Z}$-basis of $\epsilon_{I} \mathbb{Z}\left[W_{\text {ex }}\right] \epsilon_{J}$. A similar statement is true for $\mathcal{H}^{I J}$.

Theorem 3.7. The set $\left\{C_{w_{I}} \theta_{\lambda} T_{z} C_{w_{J}} \mid z \in W^{I J}, \lambda \in X(T)_{z}^{+}\right\}$is an A-basis of $\mathcal{H}^{I J}$.

This theorem is proved in $\S 7$.

Recall that $\left\{\theta_{\lambda} T_{w} \mid \lambda \in X(T), w \in W\right\}$ is the Bernstein basis of $\mathcal{H}$. By analogy, we call $\left\{C_{w_{I}} \theta_{\lambda} T_{z} C_{w_{J}} \mid z \in W^{I J}, \lambda \in X(T)_{z}^{+}\right\}$the Bernstein basis of $\mathcal{H}^{I J}$. We will see that

$$
\left\{\psi^{I J}\left(C_{w_{I}} \theta_{\lambda} T_{z} C_{w_{J}}\right) \mid z \in W^{I J}, \lambda \in X(T)_{z}^{+}\right\}
$$

is an $A$-basis of $K^{\bar{G}}\left(X^{I J}\right)$.

For $w$ in $W$ and $z$ in $W^{I J}$ define

$$
\begin{aligned}
& \mathcal{H}_{w}=\operatorname{span}_{A}\left\{\theta_{\lambda} T_{w} \mid \lambda \in X(T)\right\}, \\
& \mathcal{H}_{z}^{I J}=\operatorname{span}_{A}\left\{C_{w_{I}} \theta_{\lambda} T_{z} C_{w_{J}} \mid \lambda \in X(T)\right\}, \quad \text { and } \quad \mathcal{H}_{\leq z}^{I J}=\sum_{y \leq z} \mathcal{H}_{y}^{I J}
\end{aligned}
$$

where the last sum is over $y$ in $W^{I J}$. The proof of Theorem 3.7 shows that in fact $\left\{C_{w_{I}} \theta_{\lambda} T_{z} C_{w_{J}} \mid \lambda \in X(T)_{z}^{+}\right\}$is an $A$-basis of $\mathcal{H}_{z}^{I J}$. Extending the definition of $\mathcal{L}_{\lambda}$ for $\lambda$ in $X(T)$, let $\mathcal{L}_{\lambda}^{z}$ be the canonical $\bar{L}_{z}$-equivariant line bundle on $L_{z} / B_{z}$ determined by $\lambda$. Also, let $\chi^{\leq z}: \mathcal{H}_{\leq z} \rightarrow \mathcal{H}_{\leq z}^{I J}$ be the restriction of $\chi^{I J}$. We can now extend the diagram in Theorem 3.4 to include $\mathcal{H}_{\leq z}$ and $\mathcal{H}_{\leq z}^{I J}$. 
Theorem 3.8. Suppose $z$ is in $W^{I J}$. Then there is a commutative diagram of $A$ modules

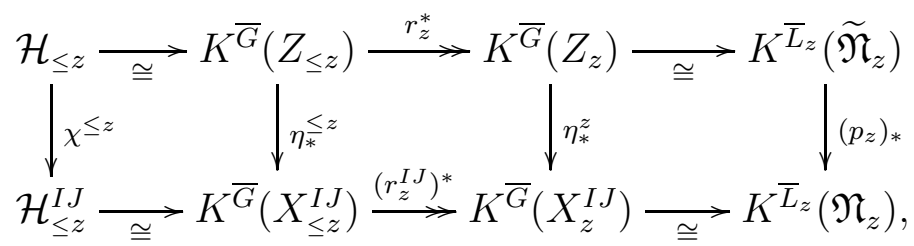

where

(1) the horizontal maps are surjective or bijective, as indicated, and the vertical maps are surjective,

(2) if $f_{1}$ is the composition across the top row, then $\mathcal{H}_{y}$ is in the kernel of $f_{1}$ for all $y<z$ and the restriction of $f_{1}$ to $\mathcal{H}_{z}$ is the isomorphism that carries $\theta_{\lambda} T_{z}$ to $\epsilon_{z} q_{z}^{*}\left[\mathcal{L}_{\lambda}^{z}\right]$ for $\lambda$ in $X(T)$, and

(3) if $f_{2}$ is the composition across the bottom row, then $\mathcal{H}_{y}^{I J}$ is in the kernel of $f_{2}$ for all $y<z$ in $W^{I J}$ and the restriction of $f_{2}$ to $\mathcal{H}_{z}^{I J}$ is the isomorphism that carries $C_{w_{I}} \theta_{\lambda} T_{z} C_{w_{J}}$ to $\epsilon_{z}\left(p_{z}\right)_{*} q_{z}^{*}\left[\mathcal{L}_{\lambda}^{z}\right]$ for $\lambda$ in $X(T)_{z}^{+}$.

This theorem is proved in $\S 8.3-\S 8.5$.

The isomorphisms in the left-hand square in (3.5) may be viewed as the restrictions of $\varphi$ and $\psi^{I J}$ to $\mathcal{H}_{\leq z}$ and $\mathcal{H}_{\leq z}^{I J}$, respectively in the sense that the inclusions $\mathcal{H}_{\leq z} \subseteq \mathcal{H}$ and $\mathcal{H}_{\leq z}^{I J} \subseteq \mathcal{H}^{I J}$, and the closed embeddings $Z_{\leq z} \subseteq Z$ and $X_{\leq z}^{I J} \subseteq X^{I J}$, induce commutative diagrams
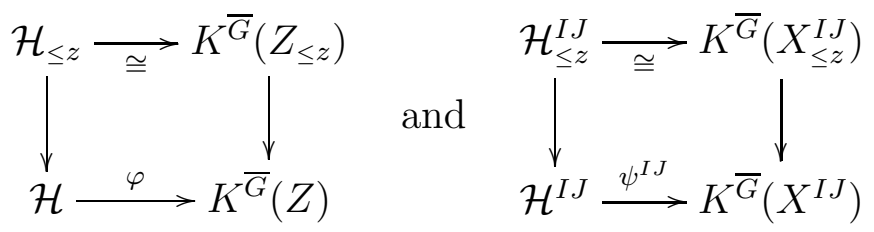

where the vertical maps are injective.

Let $z_{0}$ be the element in $W^{I J}$ with maximal length. Then $\mathcal{H}_{\leq z_{0}}^{I J}=\mathcal{H}^{I J}$ and $X_{\leq z_{0}}^{I J}=$ $X^{I J}$. Thus, taking $z=z_{0}$ in the theorem we have that $\psi^{I J}: \mathcal{H}^{I J} \rightarrow K^{\bar{G}}\left(X^{I J}\right)$ is an isomorphism. This completes the proof of Theorem 2.1.

Corollary 3.9. The set $\left\{\psi^{I J}\left(C_{w_{I}} \theta_{\lambda} T_{z} C_{w_{J}}\right) \mid z \in W^{I J}, \lambda \in X(T)_{z}^{+}\right\}$is an A-basis of $K^{\bar{G}}\left(X^{I J}\right)$.

It follows from Theorem 3.8 that $\psi^{I J}\left(\mathcal{H}_{\leq z}^{I J}\right)=K^{\bar{G}}\left(X_{\leq z}^{I J}\right)$ for $z$ in $W^{I J}$, and so the following refinement of Theorem 2.1 is a consequence of the constructions above.

Corollary 3.10. The A-submodules $K^{\bar{G}}\left(X_{\leq z}^{I J}\right)$ determine a filtration of $K^{\bar{G}}\left(X^{I J}\right)$ such that if gr $K^{\bar{G}}\left(X^{I J}\right)$ is the associated graded $A$-module, then there are A-module isomorphisms

$$
\operatorname{gr} K^{\bar{G}}\left(X^{I J}\right) \cong \bigoplus_{z \in W^{I J}} \mathcal{H}_{z}^{I J} \cong \bigoplus_{z \in W^{I J}} K^{\bar{L}_{z}}\left(\mathfrak{N}_{z}\right)
$$




\section{The "BAR" involution And Kazhdan-Lusztig Basis of $\mathcal{H}^{I J}$}

For $z$ in $W^{I J}$ and $\lambda$ in $X(T)_{z}^{+}$, let $m_{\lambda, z}$ be the element in the double coset $W_{I} t_{\lambda} z W_{J}$ with minimal length and define

$$
T_{\lambda, z}=\chi^{I J}\left(T_{m_{\lambda, z}}\right)=C_{w_{I}} T_{m_{\lambda, z}} C_{w_{J}}
$$

in $\mathcal{H}^{I J}$.

Lemma 4.1. The set $\left\{T_{\lambda, z} \mid z \in W^{I J}, \lambda \in X(T)_{z}^{+}\right\}$is an A-basis of $\mathcal{H}^{I J}$.

Proof. If $x$ is in $W_{I} t_{\lambda} z W_{J}$, then there are elements $w_{1}$ in $W_{I}$ and $w_{2}$ in $W_{J}$ so that $x=w_{1} m_{\lambda, z} w_{2}$ and $\ell(x)=\ell\left(w_{1}\right)+\ell\left(m_{\lambda, z}\right)+\ell\left(w_{2}\right)$. Thus, it follows from (2.5) that $C_{w_{I}} T_{x} C_{w_{J}}= \pm C_{w_{I}} T_{m_{\lambda, z}} C_{w_{J}}$. Therefore $\left\{T_{\lambda, z} \mid z \in W^{I J}, \lambda \in X(T)_{z}^{+}\right\}$spans $\mathcal{H}^{I J}$. By Lemma $3.6\left\{\epsilon_{I} m_{\lambda, z} \epsilon_{J} \mid z \in W^{I J}, \lambda \in X(T)_{z}^{+}\right\}$is a $\mathbb{Z}$-basis of $\epsilon_{I} \mathbb{Z}\left[W_{\text {ex }}\right] \epsilon_{J}$. It follows that $\left\{T_{\lambda, z} \mid z \in W^{I J}, \lambda \in X(T)_{z}^{+}\right\}$is linearly independent.

We call $\left\{T_{\lambda, z} \mid z \in W^{I J}, \lambda \in X(T)_{z}^{+}\right\}$the standard basis of $\mathcal{H}^{I J}$. It follows from (2.3) that the ring involution ${ }^{-}: \mathcal{H} \rightarrow \mathcal{H}$ induces an involution of abelian groups, also denoted by ${ }^{-}$, from $\mathcal{H}^{I J}$ to $\mathcal{H}^{I J}$ that we call the bar involution of $\mathcal{H}^{I J}$.

Now consider the Kazhdan-Lusztig basis $\left\{C_{x}^{\prime} \mid x \in W_{\text {ex }}\right\}$ of $\mathcal{H}$. If $t$ is in $I$ and $x$ is in $W_{\text {ex }}$ with $t x<x$, then by $(2.5)$

$$
C_{w_{I}} C_{x}^{\prime} C_{w_{J}}=-C_{w_{I}} T_{t} C_{x}^{\prime} C_{w_{J}}=-v^{2} C_{w_{I}} C_{x}^{\prime} C_{w_{J}},
$$

and so $C_{w_{I}} C_{x}^{\prime} C_{w_{J}}=0$. Similarly, if $s$ is in $J$ and $x$ is in $W_{\text {ex }}$ with $x s<x$, then $C_{w_{I}} C_{x}^{\prime} C_{w_{J}}=0$. It follows that $C_{w_{I}} C_{x}^{\prime} C_{w_{J}}=0$ unless $x=m_{\lambda, z}$ for some $\lambda, z$. For $z$ in $W^{I J}$ and $\lambda$ in $X(T)_{z}^{+}$, define

$$
C_{\lambda, z}^{\prime}=\chi^{I J}\left(C_{m_{\lambda, z}}^{\prime}\right)=C_{w_{I}} C_{m_{\lambda, z}}^{\prime} C_{w_{J}}
$$

in $\mathcal{H}^{I J}$. As usual, an element $h$ in $\mathcal{H}$ or $\mathcal{H}^{I J}$ is said to be self-dual if $\bar{h}=h$.

Proposition 4.2. The set $\left\{C_{\lambda, z}^{\prime} \mid z \in W^{I J}, \lambda \in X(T)_{z}^{+}\right\}$is a self-dual basis of $\mathcal{H}^{I J}$ and the map $\chi^{I J}: \mathcal{H} \rightarrow \mathcal{H}^{I J}$ may be identified with projection of $\mathcal{H}$ onto the span of $\left\{C_{m_{\lambda, z}}^{\prime} \mid z \in W^{I J}, \lambda \in X(T)_{z}^{+}\right\}$, that is,

$$
\chi^{I J}\left(C_{x}^{\prime}\right)= \begin{cases}C_{\lambda, z}^{\prime} & \text { if } x=m_{\lambda, z} \text { for some } z \in W^{I J}, \lambda \in X(T)_{z}^{+}, \\ 0 & \text { otherwise. }\end{cases}
$$

Proof. We need to show that $\left\{C_{\lambda, z}^{\prime} \mid z \in W^{I J}, \lambda \in X(T)_{z}^{+}\right\}$is a basis of $\mathcal{H}^{I J}$. Suppose $x$ is in $W_{\text {ex }}$, then applying $\chi^{I J}$ to both sides of the formula for $C_{x}^{\prime}$ given in (2.4) we obtain

$$
C_{w_{I}} C_{x}^{\prime} C_{w_{J}}=\sum_{y \leq x} v_{x}^{-1} P_{y, x} C_{w_{I}} T_{y} C_{w_{J}}
$$

If the left-hand side is non-zero, then it is $\chi^{I J}\left(C_{m_{\lambda, z}}^{\prime}\right)=C_{\lambda, z}^{\prime}$ for some $z$ and $\lambda$. We've observed that $C_{w_{I}} T_{y} C_{w_{J}}= \pm C_{w_{I}} T_{\lambda^{\prime}, z^{\prime}} C_{w_{J}}$ when $y$ is in $W_{I} m_{\lambda^{\prime}, z^{\prime}} W_{J}$. Moreover, it 
follows from the definition of the Bruhat order on $W_{\text {ex }}$ and [6, Lemma 2.2] that if $y<x$ and $y$ is in $W_{I} m_{\lambda^{\prime}, z^{\prime}} W_{J}$, then $m_{\lambda^{\prime}, z^{\prime}}<m_{\lambda, z}$. Therefore,

$$
C_{\lambda, z}^{\prime}=v_{m_{\lambda, z}}^{-1} T_{\lambda, z}+\sum_{m_{\lambda^{\prime}, z^{\prime}}<m_{\lambda, z}} p_{\lambda^{\prime}, z^{\prime}} T_{\lambda^{\prime}, z^{\prime}}
$$

for some polynomials $p_{\lambda^{\prime}, z^{\prime}}$ in $A$. It follows that the system of equations (4.1) indexed by pairs $(z, \lambda)$ can be solved for $\left\{T_{\lambda, z} \mid z \in W^{I J}, \lambda \in X(T)_{z}^{+}\right\}$and so $\left\{C_{\lambda, z}^{\prime} \mid z \in\right.$ $\left.W^{I J}, \lambda \in X(T)_{z}^{+}\right\}$is a basis of $\mathcal{H}^{I J}$.

We call $\left\{C_{\lambda, z}^{\prime} \mid z \in W^{I J}, \lambda \in X(T)_{z}^{+}\right\}$the Kazhdan-Lusztig basis of $\mathcal{H}^{I J}$. The elements of the Kazhdan-Lusztig basis have the standard uniqueness property, analogous to (2.4). The proof of [11, Theorem 1.1] is easily adapted to prove the following (see [18, Claim 2.4]).

Proposition 4.3. For $z$ in $W^{I J}$ and $\lambda$ in $X(T)_{z}^{+}, C_{\lambda, z}^{\prime}$ is the unique element in $\mathcal{H}^{I J}$ such that

$$
\left\{\begin{array}{l}
\overline{C_{\lambda, z}^{\prime}}=C_{\lambda, z}^{\prime} \quad \text { and } \\
C_{\lambda, z}^{\prime}=v_{m_{\lambda, z}}^{-1} T_{\lambda, z}+\sum_{m_{\lambda^{\prime}, z^{\prime}}<m_{\lambda, z}} v_{m_{\lambda, z}}^{-1} P_{\left(\lambda^{\prime}, z^{\prime}\right),(\lambda, z)} T_{\lambda^{\prime}, z^{\prime}},
\end{array}\right.
$$

where $P_{\left(\lambda^{\prime}, z^{\prime}\right),(\lambda, z)}$ is a polynomial in $v$ of degree at most $\ell\left(m_{\lambda, z}\right)-\ell\left(m_{\lambda^{\prime}, z^{\prime}}\right)-1$.

To summarize, we have the standard basis

$$
\left\{T_{\lambda, z} \mid z \in W^{I J}, \lambda \in X(T)_{z}^{+}\right\}=\left\{\chi^{I J}\left(T_{m_{\lambda, z}}\right) \mid z \in W^{I J}, \lambda \in X(T)_{z}^{+}\right\},
$$

the Bernstein basis

$$
\left\{C_{w_{I}} \theta_{\lambda} T_{z} C_{w_{J}} \mid z \in W^{I J}, \lambda \in X(T)_{z}^{+}\right\}=\left\{\chi^{I J}\left(\theta_{\lambda} T_{z}\right) \mid z \in W^{I J}, \lambda \in X(T)_{z}^{+}\right\},
$$

and the Kazhdan-Lusztig basis

$$
\left\{C_{\lambda, z}^{\prime} \mid z \in W^{I J}, \lambda \in X(T)_{z}^{+}\right\}=\left\{\chi^{I J}\left(C_{m_{\lambda, z}}^{\prime}\right) \mid z \in W^{I J}, \lambda \in X(T)_{z}^{+}\right\}
$$

of $\mathcal{H}^{I J}$. Applying $\psi^{I J}$ to each of these bases we obtain standard, Bernstein, and Kazhdan-Lusztig bases of $K^{\bar{G}}\left(X^{I J}\right)$.

\section{INTERSECTION/TOR PRODUCTS}

In this section we formalize the intersection/Tor product constructions described in $[15, \S 6.7]$ in the form they are used in this paper.

\subsection{The basic construction}

Suppose that $V, A, B$, and $C$ are $H$-varieties such that $V$ is smooth. Let $V_{1}$ and $V_{2}$ be closed, $H$-stable subvarieties of $V$ and let $p_{1}: V_{1} \rightarrow A, p_{2}: V_{2} \rightarrow B$, and $p_{12}: V_{1} \cap V_{2} \rightarrow C$ be $H$-equivariant maps such that $p_{1}$ and $p_{2}$ are smooth and $p_{12}$ is 
proper. These spaces and maps can be assembled in the commutative diagram

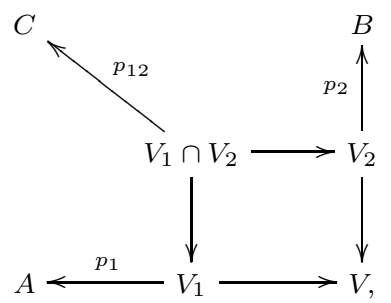

where the unlabeled maps are inclusions.

The intersection/Tor-product construction described by Lusztig [15, §6.4] can be used to define an $R(H)$-bilinear map $\star: K^{H}(A) \times K^{H}(B) \rightarrow K^{H}(C)$ as in $[15, \S 7]$. Precisely, for $\mathcal{F}$ and $\mathcal{G}$ in $K^{H}(A)$ and $K^{H}(B)$, respectively, define

$$
\mathcal{F} \star \mathcal{G}=\left(p_{12}\right)_{*}\left(p_{1}^{*} \mathcal{F} \otimes_{V}^{L} p_{2}^{*} \mathcal{G}\right) \in K^{H}(C)
$$

where the Tor-product $\otimes_{V}^{L}$ is with respect to the smooth variety $V$ and its closed subvarieties $V_{1}$ and $V_{2}$.

Next suppose that $V^{\prime}, A^{\prime}, B^{\prime}, C^{\prime}, V_{1}^{\prime}, V_{2}^{\prime}, p_{1}^{\prime}, p_{2}^{\prime}$, and $p_{12}^{\prime}$ are as in (5.1). Then we have a commutative diagram analogous to (5.1) that we connote by $(5.1)^{\prime}$, and an $R(H)$-bilinear map $\star^{\prime}: K^{H}\left(A^{\prime}\right) \times K^{H}\left(B^{\prime}\right) \rightarrow K^{H}\left(C^{\prime}\right)$.

By a morphism from (5.1) to $(5.1)^{\prime}$ we mean a tuple $(\mu, f, g, h)$ of $H$-equivariant morphisms where $\mu: V \rightarrow V^{\prime}, f: A \rightarrow A^{\prime}, g: B \rightarrow B^{\prime}$, and $h: C \rightarrow C^{\prime}$ satisfy the following conditions.

- $\mu$ is smooth, $V_{1} \subseteq \mu^{-1}\left(V_{1}^{\prime}\right)$, and $V_{2} \subseteq \mu^{-1}\left(V_{2}^{\prime}\right)$. Let $\mu_{1}, \mu_{2}$, and $\mu_{12}$ denote the restrictions of $\mu$ to maps $V_{1} \rightarrow V_{1}^{\prime}, V_{2} \rightarrow V_{2}^{\prime}$, and $V_{1} \cap V_{2} \rightarrow V_{1}^{\prime} \cap V_{2}^{\prime}$, respectively.

- $f p_{1}=p_{1}^{\prime} \mu_{1}, g p_{2}=p_{2}^{\prime} \mu_{2}$, and $h p_{12}=p_{12}^{\prime} \mu_{12}$.

Then the following diagram commutes:

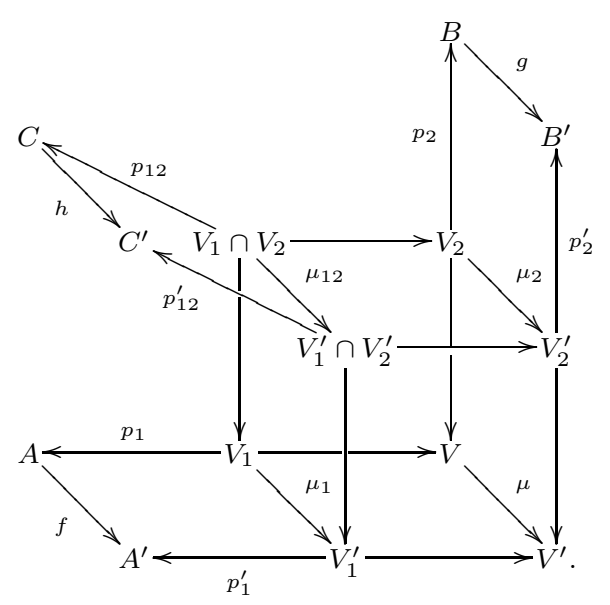


If $f$ is flat, $g$ and $h$ are proper, $V_{1}=\mu^{-1}\left(V_{1}^{\prime}\right)$, and the diagram

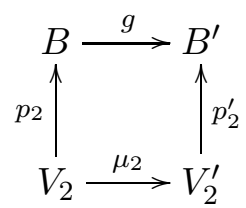

is cartesian, then $(\mu, f, g, h)$ is a proper morphism. If $f, g$, and $h$ are smooth, $V_{1}=$ $\mu^{-1}\left(V_{1}^{\prime}\right), V_{2}=\mu^{-1}\left(V_{2}^{\prime}\right)$, and the diagram

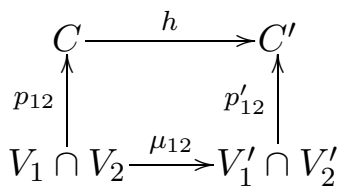

is cartesian, then $(\mu, f, g, h)$ is a smooth morphism.

The next proposition is a restatement of $[15, \S 6.5,6.6$, and 6.7].

Proposition 5.1. Suppose $\mathcal{F}^{\prime}$ is in $K^{H}\left(A^{\prime}\right), \mathcal{G}^{\prime}$ is in $K^{H}\left(B^{\prime}\right), \mathcal{F}$ is in $K^{H}(A)$, and $\mathcal{G}$ is in $K^{H}(B)$.

(1) If $(\mu, f, g, h)$ is proper, then $h_{*}\left(f^{*} \mathcal{F}^{\prime} \star \mathcal{G}\right)=\mathcal{F}^{\prime} \star^{\prime} g_{*} \mathcal{G}$ in $K^{H}\left(C^{\prime}\right)$.

(2) If $(\mu, f, g, h)$ is smooth, then $h^{*}\left(\mathcal{F}^{\prime} \star^{\prime} \mathcal{G}^{\prime}\right)=f^{*}\left(\mathcal{F}^{\prime}\right) \star g^{*}\left(\mathcal{G}^{\prime}\right)$ in $K^{H}(C)$.

(3) If $B=V_{2}=V, p_{2}=i d$, and $\mathbb{C}_{V}$ is the trivial line bundle on $V$, then $\mathcal{F} \star\left[\mathbb{C}_{V}\right]=\left(p_{12}\right)_{*} p_{1}^{*}(\mathcal{F})$ in $K^{H}(C)$.

\subsection{Applications}

In this subsection we apply the product constructions above in the setting of Steinberg varieties.

As a first application we consider the $K^{\bar{G}}(Z)$-module structure on $K^{\bar{G}}\left(X^{\emptyset J}\right)$ used in the construction of $\psi^{I J}$. If $Y$ is a subvariety of $Z$, let

$$
\widetilde{Y}=\{((x, g B),(x, h B)) \mid(x, g B, h B) \in Y\}
$$

denote the image of $Y$ in $\widetilde{\mathfrak{N}} \times \widetilde{\mathfrak{N}}$ under the natural embedding $Z \rightarrow \widetilde{\mathfrak{N}} \times \widetilde{\mathfrak{N}}$.

As in $[15, \S 7.9]$, it is easy to see that taking $V=\widetilde{\mathfrak{N}}^{3}, V_{1}=\widetilde{Z} \times \widetilde{\mathfrak{N}}, V_{2}=\widetilde{\mathfrak{N}} \times \widetilde{Z}$, and using the obvious projections $V_{1} \rightarrow Z$ and $V_{2} \rightarrow Z$ in (5.1), we obtain a "convolution product"

$$
\star: K^{\bar{G}}(Z) \times K^{\bar{G}}(Z) \rightarrow K^{\bar{G}}(Z)
$$

that defines an $R(\bar{G})$-algebra structure on $K^{\bar{G}}(Z)$.

Define $\widetilde{\mathfrak{N}}^{J}=\left\{\left(x, g P_{J}\right) \in \mathfrak{N} \times G / P_{J} \mid g^{-1} x \in \mathfrak{p}_{J}\right\}$ and

$$
\widetilde{X}^{\emptyset J}=\left\{\left((x, g B),\left(x, h P_{J}\right)\right) \in \widetilde{\mathfrak{N}} \times\left(\mathfrak{g} \times G / P_{J}\right) \mid(x, g B, h B) \in X^{\emptyset J}\right\} .
$$

Then taking $V=\widetilde{\mathfrak{N}}^{2} \times\left(\mathfrak{g} \times G / P_{J}\right), V_{1}=\widetilde{Z} \times\left(\mathfrak{g} \times G / P_{J}\right), V_{2}=\widetilde{\mathfrak{N}} \times \widetilde{X}^{\emptyset J}, p_{1}$ to be the composition $V_{1} \rightarrow \widetilde{Z} \rightarrow Z$, $p_{2}$ to be the composition $V_{2} \rightarrow \widetilde{X}^{\emptyset J} \rightarrow X^{\emptyset J}$, and defining $p_{12}: V_{1} \cap V_{2} \rightarrow X^{\emptyset J}$ by $p_{12}\left((x, g B),(x, h B),\left(x, k P_{J}\right)\right)=\left(x, g B, k P_{J}\right)$ in $(5.1)$, we obtain an $R(\bar{G})$-module linear map

$$
\star_{J}: K^{\bar{G}}(Z) \times K^{\bar{G}}\left(X^{\emptyset J}\right) \rightarrow K^{\bar{G}}\left(X^{\emptyset J}\right)
$$


that defines a $K^{\bar{G}}(Z)$-module structure on $K^{\bar{G}}\left(X^{\emptyset J}\right)$.

Define $\eta: \widetilde{\mathfrak{N}}^{3} \rightarrow \widetilde{\mathfrak{N}}^{2} \times\left(\mathfrak{g} \times G / P_{J}\right)$ by

$$
\eta((x, g B),(y, h B),(z, k B))=\left((x, g B),(y, h B),\left(z, k P_{J}\right)\right) .
$$

Then as in diagram (5.2), the tuple $\left(\eta, i d, \eta^{\emptyset J}, \eta^{\emptyset J}\right)$ defines a proper morphism from the diagram defining the multiplication on $K^{\bar{G}}(Z)$ to the diagram defining the $K^{\bar{G}}(Z)$ module structure on $K^{\bar{G}}\left(X^{\emptyset J}\right)$. The next proposition follows from Proposition $5.1(1)$.

Proposition 5.2. Suppose $J$ is a subset of $S$. There is a left $K^{\bar{G}}(Z)$-module structure on $K^{\bar{G}}\left(X^{\emptyset J}\right)$ such that $\eta_{*}^{\emptyset J}: K^{\bar{G}}(Z) \rightarrow K^{\bar{G}}\left(X^{\emptyset J}\right)$ is $K^{\bar{G}}(Z)$-linear.

As a second application we consider $K^{\bar{G}}\left(Z_{1}\right)$-module structures on $K^{\bar{G}}\left(Z_{\leq w}\right)$ and $K^{\bar{G}}\left(Z_{w}\right)$ for $w$ in $W$. Notice that $Z_{1}=\left\{(x, g B, g B) \in Z \mid g^{-1} x \in \mathfrak{u}\right\}$ is a closed subvariety of $Z$ isomorphic to $\widetilde{\mathfrak{N}}$. Thus, $Z_{1}$ is smooth and so $K^{\bar{G}}\left(Z_{1}\right)$ has a natural ring structure given by tensor product.

Suppose $w$ is in $W$. Taking $V=\widetilde{\mathfrak{N}}^{3}, V_{1}=\widetilde{Z}_{1} \times \widetilde{\mathfrak{N}}, V_{2}=\widetilde{\mathfrak{N}} \times \widetilde{Z}_{\leq w}$, and using the obvious projections $V_{1} \rightarrow Z_{1}$ and $V_{2} \rightarrow Z_{\leq w}$ in (5.1), we obtain a $K^{\bar{G}}\left(Z_{1}\right)$-module structure on $K^{\bar{G}}\left(Z_{\leq w}\right)$ that is denoted by $\star_{w}$,

$$
\star_{w}: K^{\bar{G}}\left(Z_{1}\right) \times K^{\bar{G}}\left(Z_{\leq w}\right) \rightarrow K^{\bar{G}}\left(Z_{\leq w}\right) .
$$

Notice that when $w=w_{0}$ is the longest element in $W$ we get a $K^{\bar{G}}\left(Z_{1}\right)$-module structure on $K^{\bar{G}}(Z)$. To simplify the notation slightly, set $\star_{1}=\star_{w_{0}}$. It follows from Proposition 5.1(1) that this module structure is compatible, via the inclusion $j_{1}: Z_{1} \rightarrow Z$, with the multiplication $\star$ in $K^{\bar{G}}(Z)$, in the sense that

$$
\mathcal{F} \star_{1} \mathcal{G}=\left(j_{1}\right)_{*} \mathcal{F} \star \mathcal{G}
$$

for $\mathcal{F}$ in $K^{\bar{G}}\left(Z_{1}\right)$ and $\mathcal{G}$ in $K^{\bar{G}}(Z)$.

Similarly, taking $V=\widetilde{\mathfrak{N}} \times \widetilde{Z}_{w}, V_{1}=\left(\widetilde{Z}_{1} \times \widetilde{\mathfrak{N}}\right) \cap\left(\widetilde{\mathfrak{N}} \times \widetilde{Z}_{w}\right), V_{2}=\widetilde{\mathfrak{N}} \times \widetilde{Z}_{w}$, and using the obvious projections $V_{1} \rightarrow Z_{1}$ and $V_{2} \rightarrow Z_{w}$, we obtain a $K^{\bar{G}}\left(Z_{1}\right)$-module structure on $K^{\bar{G}}\left(Z_{w}\right)$ that is denoted by $\star_{w}^{\prime}$,

$$
\star_{w}^{\prime}: K^{\bar{G}}\left(Z_{1}\right) \times K^{\bar{G}}\left(Z_{w}\right) \rightarrow K^{\bar{G}}\left(Z_{w}\right) .
$$

Now suppose $y$ and $w$ are in $W$ with $y \leq w$. Let $j_{y}^{w}: Z_{\leq y} \rightarrow Z_{\leq w}$ be the inclusion. In the special case when $w=w_{0}$ set $j_{y}=j_{y}^{w_{0}}$. Then $j_{y}$ is the inclusion of $Z_{\leq y}$ in $Z$. Recall that $r_{w}: Z_{w} \rightarrow Z_{\leq w}$ is the inclusion. Notice that $j_{y}^{w}$ is a closed embedding and that $r_{w}$ is an open embedding. The proof of the next lemma is a straightforward application of Proposition 5.1.

Lemma 5.3. Suppose $y$ and $w$ are in $W$ with $y \leq w$. Then the maps

$$
\left(j_{y}^{w}\right)_{*}: K^{\bar{G}}\left(Z_{\leq y}\right) \rightarrow K^{\bar{G}}\left(Z_{\leq w}\right) \quad \text { and } \quad r_{w}^{*}: K^{\bar{G}}\left(Z_{\leq w}\right) \rightarrow K^{\bar{G}}\left(Z_{w}\right)
$$

are $K^{\bar{G}}\left(Z_{1}\right)$-module homomorphisms. 
6. The $K^{\bar{G}}(Z)$-Module homomorphism $\eta_{*}^{\emptyset J}: K^{\bar{G}}(Z) \rightarrow K^{\bar{G}}\left(X^{\emptyset J}\right)$

In this section we prove Theorem 3.2, the assertion that $\eta_{*}^{\emptyset J}\left(\mathbf{a}_{s}\right)=0$ for $s$ in $J$, and thus complete the argument that there is an $R(\bar{G})$-module homomorphism $\psi^{I J}: \mathcal{H}^{I J} \rightarrow K^{\bar{G}}\left(X^{I J}\right)$ such that $\psi^{I J} \chi^{I J}=\eta^{I J} \varphi$ as in Theorem 2.1 .

For a subset $I$ of $S$, define $\mathcal{O}_{I}$ to be the orbit $G \cdot\left(B, w_{I} B\right)$ in $G / B \times G / B$ and define $Z_{I}=Z_{w_{I}}$ in $Z$.

Proposition 6.1. Suppose $I$ is a subset of $S$.

(1) The closure of $\mathcal{O}_{I}$ in $G / B \times G / B$ is

$$
\overline{\mathcal{O}}_{I}=\coprod_{w \in W_{I}} \mathcal{O}_{w}=\left\{(g B, h B) \mid g^{-1} h \in P_{I}\right\} .
$$

(2) The closure of $Z_{I}$ in $Z$ is

$$
\begin{aligned}
\bar{Z}_{I} & =\left\{(x, g B, h B) \in Z \mid(g B, h B) \in \overline{\mathcal{O}}_{I}, g^{-1} x \in \mathfrak{u}_{I}, h^{-1} x \in \mathfrak{u}_{I}\right\} \\
& =\left\{(x, g B, h B) \in Z \mid g^{-1} h \in P_{I}, g^{-1} x \in \mathfrak{u}_{I}\right\} .
\end{aligned}
$$

(3) There are isomorphisms $\overline{\mathcal{O}}_{I} \cong G \times{ }^{B} P_{I} / B$ and $\bar{Z}_{I} \cong G \times \times^{B}\left(\mathfrak{u}_{I} \times P_{I} / B\right)$ such that the diagram

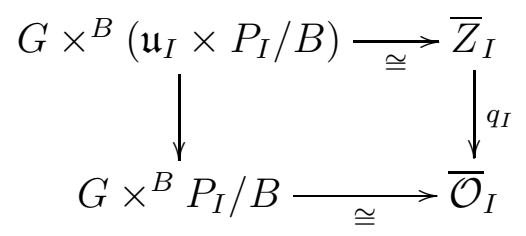

commutes. Here, the left vertical map is the obvious one and $q_{I}$ is the projection on the second and third coordinates. In particular, the varieties $\overline{\mathcal{O}}_{I}$ and $\bar{Z}_{I}$ are smooth and the projection $q_{I}: \bar{Z}_{I} \rightarrow \overline{\mathcal{O}}_{I}$ is a vector bundle with fibre $\mathfrak{u}_{I}$.

Proof. The first equality in (1) is well known. For the second equality, set

$$
Y_{1}=\left\{(g B, h B) \mid g^{-1} h \in P_{I}\right\} .
$$

Given $g$ in $G,\left(g B, g w_{I} B\right)$ is obviously in $Y_{1}$ so $\mathcal{O}_{I} \subseteq Y_{1}$. Thus $\overline{\mathcal{O}}_{I} \subseteq Y_{1}$. Conversely, if $(g B, h B)$ is in $Y_{1}$, then there is a $w$ in $W_{I}$ so that $g^{-1} h$ is in $B w B$. Say $g^{-1} h=b_{1} w b_{2}$. Then $(g B, h B)=\left(g B, g b_{1} w B\right)$ is in $\mathcal{O}_{w}$. This proves the first statement.

The second equality in (2) follows from (1). Set

$$
Y_{2}=\left\{(x, g B, h B) \in Z \mid g^{-1} h \in P_{I}, g^{-1} x \in \mathfrak{u}_{I}\right\} .
$$

Then $Y_{2}$ is closed in $Z$ and the projection $Y_{2} \rightarrow \overline{\mathcal{O}}_{I}$ is a vector bundle with fibre $\mathfrak{u}_{I}$. It follows that $Y_{2}$ is irreducible. Since

$$
Z_{I}=\left\{\left(x, g B, g w_{I} B\right) \mid g^{-1} x \in \mathfrak{u} \cap w_{I} \mathfrak{u}\right\}
$$


and $\mathfrak{u}_{I}=\mathfrak{u} \cap w_{I} \mathfrak{u}$, we see that $Z_{I}$, and hence $\bar{Z}_{I}$, is contained in $Y_{2}$. Since $\bar{Z}_{I}$ is an irreducible component of $Z$ and $Y_{2}$ is closed and irreducible we have $\bar{Z}_{I}=Y_{2}$. This proves the second statement.

For each of the varieties $\overline{\mathcal{O}}_{I}$ and $\bar{Z}_{I}$, projection on the first factor of $G / B$ is a $G$-equivariant surjection and induces $G$-equivariant isomorphisms

$$
\overline{\mathcal{O}}_{I} \cong G \times{ }^{B} P_{I} / B \quad \text { and } \quad \bar{Z}_{I} \cong G \times{ }^{B}\left(\mathfrak{u}_{I} \times P_{I} / B\right)
$$

It is straightforward to check that diagram (6.1) commutes. This proves the last statement.

Suppose $s$ is in $S$ and consider the vector bundle $q_{I}: \bar{Z}_{I} \rightarrow \overline{\mathcal{O}}_{I}$ in the case when $I=\{s\}$. Let $i_{s}: \overline{\mathcal{O}}_{\{s\}} \rightarrow G / B \times G / B$ and $k_{s}: \bar{Z}_{\{s\}} \rightarrow Z$ be the inclusions and set $q_{s}=q_{\{s\}}$. Then the diagram of $\bar{G}$-equivariant morphisms

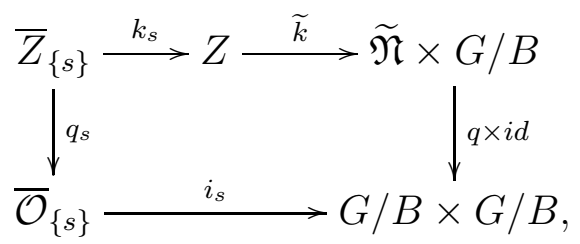

where $\widetilde{k}$ is the inclusion, commutes. Given $\lambda^{\prime}, \lambda^{\prime \prime}$ in $X(T)$, set

$$
\mathcal{L}_{\lambda^{\prime}, \lambda^{\prime \prime}}=\left(k_{s}\right)_{*} q_{s}^{*} i_{s}^{*}\left(\left[\mathcal{L}_{\lambda^{\prime}} \otimes \mathcal{L}_{\lambda^{\prime \prime}}\right]\right)=\left(k_{s}\right)_{*} k_{s}^{*} \widetilde{k}^{*}(q \times i d)^{*}\left(\left[\mathcal{L}_{\lambda^{\prime}} \otimes \mathcal{L}_{\lambda^{\prime \prime}}\right]\right)
$$

in $K^{\bar{G}}(Z)$.

Proposition 6.2. Suppose $J \subseteq S, \alpha \in \Pi_{J}, s=s_{\alpha}, \lambda^{\prime \prime} \in X(T)$ with $\left\langle\lambda^{\prime \prime}, \check{\alpha}\right\rangle=-1$, and $\lambda^{\prime} \in X(T)$. Then $\eta_{*}^{\emptyset J}\left(\left[\mathcal{L}_{\lambda^{\prime}, \lambda^{\prime \prime}}\right]\right)=0$ in $K^{\bar{G}}\left(X^{\emptyset J}\right)$.

Proof. Set $P_{s}=P_{\{s\}}, \mathfrak{u}_{s}=\mathfrak{u}_{\{s\}}$, and define

$$
Y_{s}=\eta^{\emptyset\{s\}}\left(\bar{Z}_{\{s\}}\right)=\left\{\left(x, g B, g P_{s}\right) \in \mathfrak{N} \times G / B \times G / P_{s} \mid g^{-1} x \in \mathfrak{u}_{s}\right\} .
$$

We have a commutative diagram of proper morphisms,

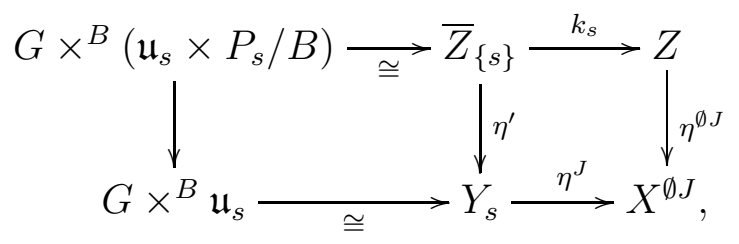

where the isomorphism $G \times{ }^{B} \mathfrak{u}_{s} \cong Y_{s}$ is induced by projection on the second factor, $\eta^{\prime}(x, g B, h B)=\left(x, g B, h P_{s}\right)$, and $\eta^{J}\left(x, g B, h P_{s}\right)=\left(x, g B, h P_{J}\right)$. Notice in particular that $\eta^{\prime}$ is a Zariski locally trivial $\mathbb{P}^{1}$-bundle.

Set $\mathcal{L}_{\lambda^{\prime}, \lambda^{\prime \prime}}^{s}=q_{s}^{*} i_{s}^{*}\left(\mathcal{L}_{\lambda^{\prime}} \otimes \mathcal{L}_{\lambda^{\prime \prime}}\right)$. To prove the proposition it is enough to show that $\eta_{*}^{\prime}\left(\left[\mathcal{L}_{\lambda^{\prime}, \lambda^{\prime \prime}}^{s}\right]\right)=0$ in $K^{\bar{G}}\left(Y_{s}\right)$. We show that $\left.R^{n} \eta_{*}^{\prime} \mathcal{L}_{\lambda^{\prime}, \lambda^{\prime \prime}}^{s}\right|_{y}=0$ for all $y$ in $Y_{s}$ and for all $n \geq 0$. It then follows that $R^{n} \eta_{*}^{\prime} \mathcal{L}_{\lambda^{\prime}, \lambda^{\prime \prime}}^{s}=0$ for all $n \geq 0$ and so $\eta_{*}^{\prime}\left(\left[\mathcal{L}_{\lambda^{\prime}, \lambda^{\prime \prime}}^{s}\right]\right)=0$ by the definition of $\eta_{*}^{\prime}$. 
Suppose $y$ is in $Y_{s}$. Because $\eta^{\prime}$ is $G$-equivariant we may assume $y=\left(x, B, P_{s}\right)$ for some $x$ in $\mathfrak{u}_{s}$. Define $E=\left(\eta^{\prime}\right)^{-1}(y)=\left\{(x, B, p B) \mid p \in P_{s}\right\}$ and let $f: E \rightarrow \bar{Z}_{\{s\}}$ be the inclusion mapping. Projection on the third factor defines an isomorphism $E \cong P_{s} / B$ and so $E \cong \mathbb{P}^{1}$.

The restriction of $\mathcal{L}_{\lambda^{\prime}, \lambda^{\prime \prime}}^{s}$ to $E$ is given by

$$
\left.\mathcal{L}_{\lambda^{\prime}, \lambda^{\prime \prime}}^{s}\right|_{E}=\left(i_{s} q_{s} f\right)^{*}\left(\mathcal{L}_{\lambda^{\prime}} \otimes \mathcal{L}_{\lambda^{\prime \prime}}\right)=\left(p_{1} i_{s} q_{s} f\right)^{*} \mathcal{L}_{\lambda^{\prime}} \otimes\left(p_{2} i_{s} q_{s} f\right)^{*} \mathcal{L}_{\lambda^{\prime \prime}}
$$

where $p_{1}$ and $p_{2}$ are the two projections of $G / B \times G / B$ onto $G / B$. The composition $p_{1} i_{s} q_{s} f$ is a constant map, so $\mathcal{L}_{\lambda^{\prime}}$ pulls back to the trivial line bundle $\mathcal{O}_{\mathbb{P}^{1}}(0)$ on $E$. The map $p_{2} i_{s} q_{s} f$ restricts to an isomorphism $E \cong P_{s} / B$, and because $\left\langle\lambda^{\prime \prime}, \check{\alpha}\right\rangle=-1, \mathcal{L}_{\lambda^{\prime \prime}}$ pulls back to the tautological bundle on $P_{s} / B$. Therefore, $\left.\mathcal{L}_{\lambda^{\prime}, \lambda^{\prime \prime}}^{s}\right|_{E}$ may be identified with the line bundle $\mathcal{O}_{\mathbb{P}^{1}}(-1)$ on $\mathbb{P}^{1}$.

Finally, for $n \geq 0, H^{n}\left(E,\left.\mathcal{L}_{\lambda^{\prime}, \lambda^{\prime \prime}}^{s}\right|_{E}\right) \cong H^{n}\left(\mathbb{P}^{1}, \mathcal{O}_{\mathbb{P}^{1}}(-1)\right)=0$, and hence by Grauert's Theorem, $\left.R^{n} \eta_{*}^{\prime} \mathcal{L}_{\lambda^{\prime}, \lambda^{\prime \prime}}^{s}\right|_{y} \cong H^{n}\left(E,\left.\mathcal{L}_{\lambda^{\prime}, \lambda^{\prime \prime}}^{s}\right|_{E}\right)=0$ as claimed.

We can now complete the proof of Theorem 3.2. With $J, \alpha$, and $s$ as above, suppose that $\lambda^{\prime}$ and $\lambda^{\prime \prime}$ are in $X(T),\left\langle\lambda^{\prime}, \check{\alpha}\right\rangle=\left\langle\lambda^{\prime \prime}, \check{\alpha}\right\rangle=-1$, and $-\alpha=\lambda^{\prime}+\lambda^{\prime \prime}$. Define $\mathbf{a}_{s}$ in $K^{\bar{G}}(Z)$ by

$$
\mathbf{a}_{s}=v^{-1} \mathcal{L}_{\lambda^{\prime}, \lambda^{\prime \prime}}=v^{-1}\left(k_{s}\right)_{*} q_{s}^{*} i_{s}^{*}\left(\left[\mathcal{L}_{\lambda^{\prime}} \otimes \mathcal{L}_{\lambda^{\prime \prime}}\right]\right) .
$$

Lusztig $[15, \S 7.19,7.25]$ has shown that $\mathbf{a}_{s}$ does not depend on the choice of $\lambda^{\prime}$ and $\lambda^{\prime \prime}$. It follows from Proposition 6.2 that $\eta_{*}^{\emptyset J}\left(\mathbf{a}_{s}\right)=0$, as asserted in the statement of the theorem.

\section{AN $A$-BASIS OF $\mathcal{H}^{I J}$}

By Lemma 3.6, $\left\{t_{\lambda} z \mid z \in W^{I J}, \lambda \in X(T)_{z}^{+}\right\}$is a set of $\left(W_{I}, W_{J}\right)$-double coset representatives in $W_{\text {ex }}$. In this section we prove Theorem 3.7 and thus show that $\mathcal{H}^{I J}$ has an $A$-basis indexed by $W_{I} \backslash W_{\mathrm{ex}} / W_{J}$.

We need to show that the set $\mathcal{E}=\left\{C_{w_{I}} \theta_{\lambda} T_{z} C_{w_{J}} \mid z \in W^{I J}, \lambda \in X(T)_{z}^{+}\right\}$is an $A$-basis of $\mathcal{H}^{I J}$. To see that $\mathcal{E}$ is linearly independent, notice that the specialization $v \mapsto 1$ defines an $A$-linear homomorphism

$$
\mathcal{H}^{I J} \rightarrow \epsilon_{I} \mathbb{Z}\left[W_{\mathrm{ex}}\right] \epsilon_{J}
$$

where as above, $\epsilon_{I}=\sum_{w \in W_{I}} \epsilon_{w} w$. By Lemma 3.6, $\left\{\epsilon_{I} t_{\lambda} z \epsilon_{J} \mid z \in W^{I J}, \lambda \in X(T)_{z}^{+}\right\}$ is linearly independent in $\epsilon_{I} \mathbb{Z}\left[W_{\text {ex }}\right] \epsilon_{J}$ and so $\mathcal{E}$ is linearly independent in $\mathcal{H}^{I J}$.

To prove that $\mathcal{E}$ spans $\mathcal{H}^{I J}$ we adapt an argument of Nelsen and Ram [16]. It follows from the relation

$$
\theta_{\lambda} T_{s}-T_{s} \theta_{s(\lambda)}=\left(v^{2}-1\right) \frac{\theta_{\lambda}-\theta_{s(\lambda)}}{1-\theta_{-\alpha}},
$$

for $\lambda$ in $X(T)$ and $s=s_{\alpha}$ with $\alpha$ in $\Pi$ that if $w$ is in $W$, then $\theta_{\lambda} T_{w}$ is in the $A$-span of $\left\{T_{y} \theta_{\mu} \mid y \leq w, \mu \in X(T)\right\}$. Therefore, if $w$ is in $W$ and $w=w_{1} z w_{2}$ where $z$ is in $W^{I J}$, $w_{1}$ in $W_{I}, w_{2}$ in $W_{J}$, and $\ell(w)=\ell\left(w_{1}\right)+\ell(z)+\ell\left(w_{2}\right)$, then $\theta_{\lambda} T_{w_{1} z w_{2}}=\theta_{\lambda} T_{w_{1}} T_{z} T_{w_{2}}$ is an $A$-linear combination of elements of the form $T_{y} \theta_{\mu} T_{z} T_{w_{2}}$ where $y \leq w_{1}$ is in $W_{I}$. Therefore, the elements $C_{w_{I}} \theta_{\lambda} T_{z} C_{w_{J}}$ for $z$ in $W^{I J}$ and $\lambda$ in $X(T)$ span $\mathcal{H}^{I J}$. 
Fix $z$ in $W^{I J}$ and suppose that $s=s_{\alpha}$ with $\alpha$ in $\Pi_{I} \cap z \Pi_{J}$. Then $T_{s} T_{z}=T_{s z}=$ $T_{z z^{-1} s z}=T_{z} T_{s^{\prime}}$, where $s^{\prime}=z^{-1} s z$ is in $J$. Thus, multiplying (7.1) on the right by $T_{z}$ and then taking the image in $\mathcal{H}^{I J}$, we have

$$
C_{w_{I}}\left(-\theta_{\lambda} T_{z}+\theta_{s(\lambda)} T_{z}\right) C_{w_{J}}=C_{w_{I}}\left(\left(v^{2}-1\right) \frac{\theta_{\lambda}-\theta_{s(\lambda)}}{1-\theta_{-\alpha}} T_{z}\right) C_{w_{J}}
$$

for any $\lambda$ in $X(T)$. Replacing $\lambda$ by $\lambda-\alpha$, subtracting the result from (7.2), and simplifying, we get

$$
C_{w_{I}} \theta_{s(\lambda)} T_{z} C_{w_{J}}=C_{w_{I}}\left(v^{2} \theta_{\lambda}-\theta_{\lambda-\alpha}+v^{2} \theta_{s(\lambda)+\alpha}\right) T_{z} C_{w_{J}}
$$

The derivation of (7.3) from (7.1) is the same as in the proof of [16, Proposition 2.1]. After relabeling the elements in $\mathcal{H}$ and replacing $t$ by $v^{2},(7.3)$ is the same as the recursion formula at the end of the proof of [16, Proposition 2.1]. It follows that if $\lambda$ is in $X(T)$ with $d=\langle\lambda, \check{\alpha}\rangle \geq 0$, then there are polynomials $p_{0}, p_{1}, \ldots, p_{\lfloor d / 2\rfloor}$ in $\mathbb{Z}\left[v^{2}\right]$ such that

$$
C_{w_{I}} \theta_{s(\lambda)} T_{z} C_{w_{J}}=C_{w_{I}}\left(\sum_{j=0}^{\lfloor d / 2\rfloor} p_{j} \theta_{\lambda-j \alpha}\right) T_{z} C_{w_{J}}
$$

The polynomials $p_{j}$ do not depend on $\lambda$ and are given by the formula in [16, Proposition 2.1] with $t$ replaced by $v^{2}$.

Now if $\mu$ is in $X(T)$ and $\langle\mu, \check{\alpha}\rangle<0$, then using (7.4) with $s(\lambda)=\mu$ we see that $C_{w_{I}} \theta_{\mu} T_{z} C_{w_{J}}$ may be rewritten as an $A$-linear combination of terms $C_{w_{I}} \theta_{\mu^{\prime}} T_{z} C_{w_{J}}$ where $\left\langle\mu^{\prime}, \check{\alpha}\right\rangle \geq 0$ and $\left\langle\mu^{\prime}, \check{\beta}\right\rangle \geq\langle\mu, \check{\beta}\rangle$ for all $s_{\beta}$ with $\beta$ in $\Pi$. It follows that $\mathcal{E}$ spans $\mathcal{H}^{I J}$, as claimed.

\section{Equivariant $K$-THEORY OF $X^{I J}$}

In this section we compute $K^{\bar{G}}\left(X^{I J}\right)$ in terms of $\mathcal{H}^{I J}$, using $\mathcal{H}$ and $K^{\bar{G}}(Z)$, and thus complete the proof of Theorem 2.1. We begin with $K^{\bar{G}}\left(X_{z}^{I J}\right)$.

8.1. $K^{\bar{G}}\left(X_{z}^{I J}\right)$ and the Springer resolution of $\mathfrak{N}_{z}$

In this subsection we prove Theorem 3.4.

Suppose $z$ is in $W^{I J}$. We need to show that there is a commutative diagram of $R(\bar{G})$-modules

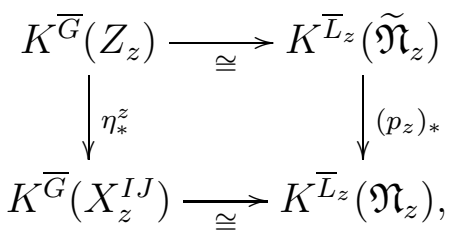

where the horizontal maps are isomorphisms, $p_{z}$ is the Springer resolution of the nilpotent cone $\mathfrak{N}_{z}$ of $\mathfrak{l}_{z}$, and the vertical maps are surjections.

Set

$$
P_{z}=P_{I} \cap{ }^{z} P_{J} \quad \text { and } \quad V_{z}=\left(L_{I} \cap{ }^{z} U_{J}\right)\left(U_{I} \cap{ }^{z} L_{J}\right)\left(U_{I} \cap{ }^{z} U_{J}\right) .
$$


Then

$$
P_{z}=L_{z} V_{z}=L_{z}\left(L_{I} \cap{ }^{z} U_{J}\right)\left(U_{I} \cap{ }^{z} L_{J}\right)\left(U_{I} \cap{ }^{z} U_{J}\right)
$$

$L_{z}$ is a Levi subgroup of $P_{z}$, and $V_{z}$ is the unipotent radical of $P_{z}$ (see [5, §69B]). The Lie algebra analog of the factorization (8.1) is

$$
\mathfrak{p}_{z}=\mathfrak{l}_{z}+\mathfrak{v}_{z}=\mathfrak{l}_{z}+\left(\mathfrak{l}_{I} \cap z \mathfrak{u}_{J}\right)+\left(\mathfrak{u}_{I} \cap z \mathfrak{l}_{J}\right)+\left(\mathfrak{u}_{I} \cap z \mathfrak{u}_{J}\right) .
$$

Set $\mathfrak{u}_{z}=\mathfrak{u} \cap \mathfrak{l}_{z}$. It follows from (8.2) that $\mathfrak{u} \cap z \mathfrak{u}=\mathfrak{u}_{z}+\mathfrak{v}_{z}$.

The rule $(x, g B, g z B) \mapsto\left(x, g P_{I}, g z P_{J}\right)$ defines a surjective, $G$-equivariant morphism from $Z_{z}$ onto the $G$-orbit of $\left(P_{I}, z P_{J}\right)$ in $G / P_{I} \times G / P_{J}$. The fibre over $\left(P_{I}, z P_{J}\right)$ is

$$
\begin{aligned}
F & =\left\{(x, p B, p z B) \in Z_{z} \mid p \in P_{z}, p^{-1} x \in \mathfrak{u} \cap z \mathfrak{u}\right\} \\
& =\left\{(x, h B, h z B) \in Z_{z} \mid h \in L_{z}, h^{-1} x \in \mathfrak{u}_{z}+\mathfrak{v}_{z}\right\} .
\end{aligned}
$$

Thus, $Z_{z} \cong G \times{ }^{P_{z}} F$. Projection onto the second and third factors is a surjective, $P_{z}$-equivariant morphism from $F$ onto the $P_{z}$-orbit of $(B, z B)$ in $G / B \times G / B$ with fibre over $(B, z B)$ isomorphic to $\mathfrak{u}_{z}+\mathfrak{v}_{z}$. Thus, $F \cong P_{z} \times{ }^{B \cap^{z} B}\left(\mathfrak{u}_{z}+\mathfrak{v}_{z}\right)$. Define

$$
\tilde{p}: F \rightarrow \widetilde{\mathfrak{N}}_{z} \quad \text { by } \quad \tilde{p}(h(x+v), h B, h z B)=\left(h x, h B_{z}\right),
$$

for $x$ in $\mathfrak{u}_{z}, v$ in $\mathfrak{v}_{z}$, and $h$ in $L_{z}$, and define

$$
i_{z}: \widetilde{\mathfrak{N}}_{z} \rightarrow F \quad \text { by } \quad i_{z}\left(x, h B_{z}\right)=(x, h B, h z B) .
$$

Then $\tilde{p}$ and $i_{z}$ are well-defined morphisms that may be identified with the morphisms $P_{z} \times{ }^{B \cap^{z} B}\left(\mathfrak{u}_{z}+\mathfrak{v}_{z}\right) \rightarrow L_{z} \times{ }^{B_{z}} \mathfrak{u}_{z}$ and $L_{z} \times{ }^{B_{z}} \mathfrak{u}_{z} \rightarrow P_{z} \times{ }^{B \cap^{z} B}\left(\mathfrak{u}_{z}+\mathfrak{v}_{z}\right)$ given by $h u *(x+v) \mapsto h * x$ and $h * x \mapsto h * x$ for $h$ in $L_{z}, u$ in $V_{z}, x$ in $\mathfrak{u}_{z}$, and $v$ in $\mathfrak{v}_{z}$. Thus, $\tilde{p}: F \rightarrow \widetilde{\mathfrak{N}}_{z}$ is an $\bar{L}_{z}$-equivariant vector bundle with fibre $\mathfrak{v}_{z}$ and zero section $i_{z}$.

Next, the projection of $X_{z}^{I J}$ onto the second and third factors is a surjective, $G$ equivariant morphism onto the $G$-orbit of $\left(P_{I}, z P_{J}\right)$ in $G / P_{I} \times G / P_{J}$. The fibre over $\left(P_{I}, z P_{J}\right)$ is

$$
F^{I J}=\left\{\left(x, P_{I}, z P_{J}\right) \in X_{z}^{I J} \mid x \in \mathfrak{N}_{z}+\mathfrak{v}_{z}\right\}
$$

Thus, $X_{z}^{I J} \cong G \times^{P_{z}}\left(\mathfrak{N}_{z}+\mathfrak{v}_{z}\right)$ and $F^{I J} \cong \mathfrak{N}_{z}+\mathfrak{v}_{z}$. Define

$$
\tilde{p}^{I J}: F^{I J} \rightarrow \mathfrak{N}_{z} \quad \text { by } \quad \tilde{p}^{I J}\left(x+v, P_{I}, z P_{J}\right)=x,
$$

for $x$ in $\mathfrak{N}_{z}$ and $v$ in $\mathfrak{v}_{z}$, and define

$$
i_{z}^{I J}: \mathfrak{N}_{z} \rightarrow F^{I J} \quad \text { by } \quad i_{z}^{I J}(x)=\left(x, P_{I}, z P_{J}\right)
$$

Obviously the morphism $\tilde{p}^{I J}$ is an $\bar{L}_{z}$-equivariant vector bundle with fibre $\mathfrak{v}_{z}$ and zero section $i_{z}^{I J}$.

Finally, let $\tilde{\eta}^{z}: F \rightarrow F^{I J}$ be the restriction of $\eta^{z}$. Then the diagram

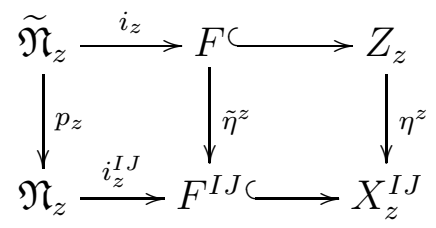


commutes. Moreover, one checks that the left-hand square is cartesian.

Now applying $K^{\bar{G}}$ and $K^{\bar{L}_{z}}$ to $(8.3)$ we get

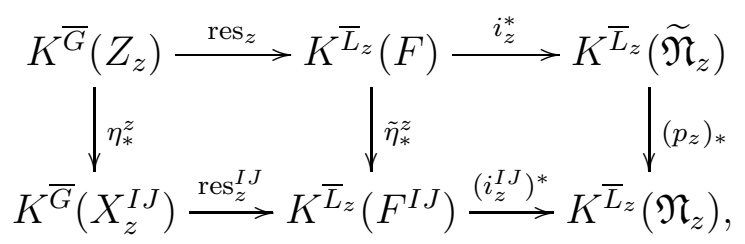

where $\operatorname{res}_{z}=\operatorname{res}_{F}$ is defined using the isomorphism $Z_{z} \cong G \times{ }^{P z} F$ and $\operatorname{res}_{z}^{I J}=\operatorname{res}_{F^{I J}}$ is defined using the isomorphism $X_{z}^{I J} \cong G \times{ }^{P_{z}} F^{I J}$. The left-hand square commutes by the naturality of res. For the right-hand square, the diagram

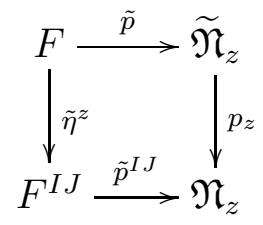

is cartesian and $\tilde{p}$ and $\tilde{p}^{I J}$ are vector bundles, so $\tilde{\eta}_{*}^{z} \tilde{p}^{*}=\left(\tilde{p}^{I J}\right)^{*}\left(p_{z}\right)_{*}$. By the Thom isomorphism in equivariant $K$-theory, $i_{z}^{*}=\left(\tilde{p}^{*}\right)^{-1}$ and $\left(i_{z}^{I J}\right)^{*}=\left(\left(\tilde{p}^{I J}\right)^{*}\right)^{-1}$, so $\left(i_{z}^{I J}\right)^{*} \tilde{\eta}_{*}^{z}=$ $\left(p_{z}\right)_{*} i_{z}^{*}$. Finally, by Lemma $2.2\left(p_{z}\right)_{*}$ is a surjection. Therefore, $\tilde{\eta}_{*}^{z}$ and $\eta_{*}^{z}$ are surjections as well.

\subsection{The sequence (3.1) is exact}

Proposition 8.1. Suppose $H$ is a linear algebraic group and that $Y$ is an $H$-variety such that $H$ acts on $Y$ with finitely many orbits. Then $K_{1}^{H}(Y)=0$.

Proof. If $H$ acts transitively with point stabilizer $H_{0}$, then $Y \cong H / H_{0}$ and the result is known (see $[12, \S 1.3(\mathrm{p})]$ ). In the general case, choose an open orbit $\mathcal{O}$ in $Y$. Then there is an exact sequence

$$
\cdots \rightarrow K_{1}^{H}(Y \backslash \mathcal{O}) \rightarrow K_{1}^{H}(Y) \rightarrow K_{1}^{H}(\mathcal{O}) \rightarrow \cdots
$$

By induction on the number of orbits, $K_{1}^{H}(Y \backslash \mathcal{O})=0$. We have already observed that $K_{1}^{H}(\mathcal{O})=0$. Thus, $K_{1}^{H}(Y)=0$.

Lemma 8.2. Suppose $I, J \subseteq S$, and $z \in W^{I J}$. Then $K_{1}^{\bar{G}}\left(X_{z}^{I J}\right)=0$.

Proof. The constructions used in the proof of Theorem 3.4 apply to the functors $K_{i}^{\bar{G}}$ for $i \geq 0$ (see $[2, \S 5.2,5.4])$ and give isomorphisms

$$
K_{1}^{\bar{G}}\left(X_{z}^{I J}\right) \stackrel{\operatorname{res}_{z}^{I J}}{\cong} K_{1}^{\bar{L}_{z}}\left(F^{I J}\right) \stackrel{\left(i_{z}^{I J}\right)^{*}}{\cong} K_{1}^{\bar{L}_{z}}\left(\mathfrak{N}_{z}\right)
$$

Because $\bar{L}_{z}$ acts on $\mathfrak{N}_{z}$ with finitely many orbits, it follows from Proposition 8.1 that $K_{1}^{\overline{L_{z}}}\left(\mathfrak{N}_{z}\right)=0$.

The fact that sequence (3.1) is exact follows immediately from Lemma 8.2 and the long exact sequence in equivariant $K$-theory. 
8.3. The isomorphism $\mathcal{H}_{\leq z}^{I J} \cong K^{\bar{G}}\left(X_{\leq z}^{I J}\right)$

The rest of this section is devoted to the proof of Theorem 3.8. We first define the maps in (3.5),

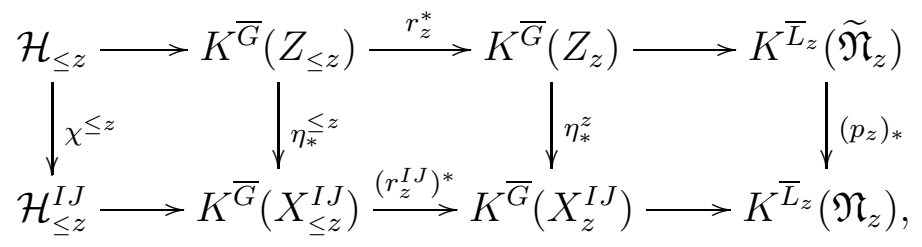

and show that the diagram commutes.

The middle square is induced by the cartesian diagram (3.3) and the right-hand square is as in Theorem 3.4. Both of these squares commute.

8.4. The left-hand square in diagram (3.5)

We observed after (7.1) that if $\lambda$ is in $X(T), y$ is in $W^{I J}$, and $w$ is in the double coset $W_{I} y W_{J}$, then $C_{w_{I}} \theta_{\lambda} T_{w} C_{w_{J}}$ is in the span of $\left\{C_{w_{I}} \theta_{\mu} T_{y} C_{w_{J}} \mid \mu \in X(T)\right\}$. Therefore, $\chi^{I J}\left(\mathcal{H}_{w}\right) \subseteq \mathcal{H}_{y}^{I J}$. It follows that $\chi^{\leq z}\left(\mathcal{H}_{\leq z}\right) \subseteq \mathcal{H}_{\leq z}^{I J}$. In particular, $\chi^{\leq z}: \mathcal{H}_{\leq z} \rightarrow \mathcal{H}_{\leq z}^{I J}$ is defined.

Consider the commutative diagram

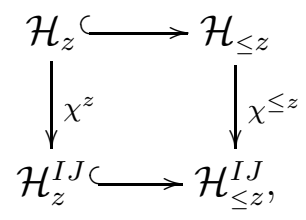

where the horizontal maps are the inclusions and $\chi^{z}$ is the restriction of $\chi^{I J}$ to $\mathcal{H}_{z}$. It is clear that $\chi^{y}\left(\mathcal{H}_{y}\right)=\mathcal{H}_{y}^{I J}$ for $y$ in $W^{I J}$ and so $\chi^{\leq z}$ is surjective.

The left-hand square in diagram (3.5) is

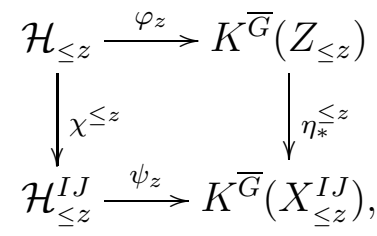

where $\varphi_{z}$ and $\psi_{z}$ are defined below.

Recall that for $w$ in $W j_{w}: Z_{\leq w} \rightarrow Z$ is the inclusion. Similarly, let $j_{z}^{I J}$ denote the inclusion $X_{\leq z}^{I J} \rightarrow X^{I J}$ for $z$ in $W^{I J}$. The maps $\varphi_{z}$ and $\psi_{z}$ are the restrictions of $\varphi$ and $\psi^{I J}$, respectively, in the sense that $\left(j_{z}\right)_{*} \circ \varphi_{z}$ is the restriction of $\varphi$ to $\mathcal{H}_{\leq z}$ and $\left(j_{z}^{I J}\right)_{*} \circ \psi_{z}$ is the restriction of $\psi^{I J}$ to $\mathcal{H}_{\leq z}^{I J}$. In order to prove Theorem 3.8 we need a formula for $\varphi_{z}$, and so we define $\varphi_{z}$ explicitly, show that $\left(j_{z}\right)_{*} \circ \varphi_{z}$ is the restriction of $\varphi$ to $\mathcal{H}_{\leq z}$, and then define $\psi_{z}$.

For $w$ in $W$, let $q_{w, 1}: Z_{w} \rightarrow G / B$ by $q_{w, 1}(x, g B, g w B)=g B$. Then $q_{w, 1}$ is a $\bar{G}$ equivariant affine space bundle over $G / B$ and so $q_{w, 1}^{*}: K^{\bar{G}}(G / B) \rightarrow K^{\bar{G}}\left(Z_{w}\right)$ is an $R(\bar{G})$-module isomorphism. 
Theorem 8.3. Suppose $w$ is in $W$. There is an A-module isomorphism

$$
\varphi_{w}: \mathcal{H}_{\leq w} \rightarrow K^{\bar{G}}\left(Z_{\leq w}\right)
$$

such that

(1) $\left(j_{w}\right)_{*} \varphi_{w}: \mathcal{H}_{\leq w} \rightarrow K^{\bar{G}}(Z)$ is the restriction of $\varphi$ to $\mathcal{H}_{\leq w}$, and

(2) for $\lambda$ in $X(T), r_{w}^{*} \varphi_{w}\left(\theta_{\lambda} T_{w}\right)=\epsilon_{w} q_{w, 1}^{*}\left[\mathcal{L}_{\lambda}\right]$.

In particular, $\left\{r_{w}^{*} \varphi_{w}\left(\theta_{\lambda} T_{w}\right) \mid \lambda \in X(T)\right\}$ is an $A$-basis of $K^{\bar{G}}\left(Z_{w}\right)$.

Proof. Lusztig has shown (see [15, Lemma 8.9]) that there is a unique element $\xi_{y}$ in $K^{\bar{G}}\left(Z_{\leq y}\right)$ such that $\left(j_{y}\right)_{*}\left(\xi_{y}\right)=\varphi\left(T_{y}\right)$. Let $d_{1}: \widetilde{\mathfrak{N}} \rightarrow Z_{1}$ be the "diagonal" isomorphism given by $d_{1}(x, g B)=(x, g B, g B)$. With this notation, using the $K^{\bar{G}}\left(Z_{1}\right)$-module structure $\star_{w}$ on $K^{\bar{G}}\left(Z_{\leq w}\right)$, define

$$
\varphi_{w}: \mathcal{H}_{\leq w} \rightarrow K^{\bar{G}}\left(Z_{\leq w}\right) \quad \text { by } \quad \varphi_{w}\left(\theta_{\lambda} T_{y}\right)=\left(d_{1}\right)_{*} q^{*}\left[\mathcal{L}_{\lambda}\right] \star_{w}\left(j_{y}^{w}\right)_{*}\left(\xi_{y}\right)
$$

for $\lambda$ in $X(T)$ and $y$ in $W$ with $y \leq w$.

Set $d=j_{1} d_{1}: \widetilde{\mathfrak{N}} \rightarrow Z$, so $d(x, g B)=(x, g B, g B)$. By the definition of $\varphi$, for $\lambda$ in $X(T), \varphi\left(\theta_{\lambda}\right)=d_{*} q^{*}\left[\mathcal{L}_{\lambda}\right]$. Thus, using equation (5.3) and Lemma 5.3 we have

$$
\begin{aligned}
\left(j_{w}\right)_{*} \varphi_{w}\left(\theta_{\lambda} T_{y}\right) & =\left(j_{w}\right)_{*}\left(\left(d_{1}\right)_{*} q^{*}\left[\mathcal{L}_{\lambda}\right] \star_{w}\left(j_{y}^{w}\right)_{*} \xi_{y}\right) \\
& =\left(j_{1}\right)_{*}\left(d_{1}\right)_{*} q^{*}\left[\mathcal{L}_{\lambda}\right] \star\left(j_{w}\right)_{*}\left(j_{y}^{w}\right)_{*} \xi_{y}=d_{*} q^{*}\left[\mathcal{L}_{\lambda}\right] \star\left(j_{y}\right)_{*} \xi_{y} \\
& =\varphi\left(\theta_{\lambda}\right) \star \varphi\left(T_{y}\right)=\varphi\left(\theta_{\lambda} T_{y}\right) .
\end{aligned}
$$

This proves the first statement.

To prove (2), let $\mathbb{C}_{Z_{w}}$ be the trivial line bundle on $Z_{w}$ and let

$$
p_{w, 1}: Z_{w} \rightarrow \widetilde{\mathfrak{N}} \quad \text { by } \quad p_{w, 1}(x, g B, g w B)=(x, g B) .
$$

Then $q_{w, 1}=q p_{w, 1}$. Set $V_{w}=\left(\widetilde{Z}_{1} \times \widetilde{\mathfrak{N}}\right) \cap\left(\widetilde{\mathfrak{N}} \times \widetilde{Z}_{w}\right)$ and let $p_{12}^{\prime}: V_{w} \rightarrow Z_{1}$ and $p_{13}^{\prime}: V_{w} \rightarrow Z_{w}$ be the obvious projections. It is straightforward to check that $p_{w, 1}$ and $p_{12}^{\prime}$ are smooth, that $p_{13}^{\prime}$ is an isomorphism, and that the diagram

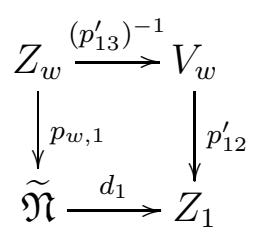

is cartesian. Thus $\left(p_{12}^{\prime}\right)^{*}\left(d_{1}\right)_{*}=\left(p_{13}^{\prime}\right)_{*}^{-1} p_{w, 1}^{*}$. Using the $K^{\bar{G}}\left(Z_{1}\right)$-module structure $\star_{w}^{\prime}$ 
on $K^{\bar{G}}\left(Z_{w}\right)$, we have

$$
\begin{aligned}
r_{w}^{*} \varphi_{w}\left(\theta_{\lambda} T_{w}\right) & =r_{w}^{*}\left(\left(d_{1}\right)_{*} q^{*}\left[\mathcal{L}_{\lambda}\right] \star_{w} \xi_{w}\right) & & \\
& =\left(d_{1}\right)_{*} q^{*}\left[\mathcal{L}_{\lambda}\right] \star_{w}^{\prime} r_{w}^{*}\left(\xi_{w}\right) & & \text { Lemma 5.3 } \\
& =\left(d_{1}\right)_{*} q^{*}\left[\mathcal{L}_{\lambda}\right] \star_{w}^{\prime} \epsilon_{w}\left[\mathbb{C}_{Z_{w}}\right] & & {[15, \S 8.9] } \\
& =\epsilon_{w}\left(p_{13}^{\prime}\right)_{*}\left(p_{12}^{\prime}\right)^{*}\left(d_{1}\right)_{*} q^{*}\left[\mathcal{L}_{\lambda}\right] & & \text { Proposition 5.1(3) } \\
& =\epsilon_{w} p_{w, 1}^{*} q^{*}\left[\mathcal{L}_{\lambda}\right] & & \\
& =\epsilon_{w} q_{w, 1}^{*}\left[\mathcal{L}_{\lambda}\right] & &
\end{aligned}
$$

This completes the proof of the second statement.

The last statement in the theorem follows from (2) and the fact that $q_{w, 1}^{*}$ is an isomorphism.

Choose a linear order on the interval $[1, w]$ in the Bruhat poset of $W$ that extends the Bruhat order. This linear order determines gradings on $\mathcal{H}_{\leq w}$ and $K^{\bar{G}}\left(Z_{\leq w}\right)$. As $\left\{\theta_{\lambda} T_{y} \mid \lambda \in X(T)\right\}$ is an $A$-basis of $\mathcal{H}_{y}$ and $\left\{r_{y}^{*} \varphi_{y}\left(\theta_{\lambda} T_{y}\right) \mid \lambda \in X(T)\right\}$ is an $A$-basis of $K^{\bar{G}}\left(Z_{y}\right)$ for $y$ in $W$, the associated graded map gr $\varphi_{w}$ is an isomorphism. Thus, $\varphi_{w}$ is an isomorphism.

It follows from the theorem that for $z$ in $W^{I J}$,

$$
\eta_{*}^{I J} \varphi\left(\mathcal{H}_{\leq z}\right)=\eta_{*}^{I J}\left(j_{z}\right)_{*}\left(K^{\bar{G}}\left(Z_{\leq z}\right)\right)=\left(j_{z}^{I J}\right)_{*} \eta_{*}^{\leq z}\left(K^{\bar{G}}\left(Z_{\leq z}\right)\right) \subseteq\left(j_{z}^{I J}\right)_{*}\left(K^{\bar{G}}\left(X_{\leq z}^{I J}\right)\right)
$$

On the other hand, we have seen that $\eta_{*}^{I J} \varphi=\psi^{I J} \chi^{I J}$ and that $\chi^{\leq z}$ is surjective, so

$$
\eta_{*}^{I J} \varphi\left(\mathcal{H}_{\leq z}\right)=\psi^{I J} \chi^{I J}\left(\mathcal{H}_{\leq z}\right)=\psi^{I J} \chi^{\leq z}\left(\mathcal{H}_{\leq z}\right)=\psi^{I J}\left(\mathcal{H}_{\leq z}^{I J}\right)
$$

Therefore, $\psi^{I J}\left(\mathcal{H}_{\leq z}^{I J}\right) \subseteq\left(j_{z}^{I J}\right)_{*}\left(K^{\bar{G}}\left(X_{\leq z}^{I J}\right)\right)$ and so there is an $A$-module homomorphism $\psi_{z}: \mathcal{H}_{\leq z}^{I J} \rightarrow K^{\bar{G}}\left(X_{\leq z}^{I J}\right)$ such that $\eta_{*}^{\leq z} \varphi_{z}=\psi_{z} \chi^{\leq z}$. In particular, the maps $\varphi_{z}$ and $\psi_{z}$ in the left-hand square in (3.5) are defined and the diagram commutes.

\subsection{Proof of Theorem 3.8}

In this subsection we complete the proof of Theorem 3.8. The arguments above show that diagram (3.5) commutes.

To prove Theorem $3.8(2)$, let $f_{1}: \mathcal{H}_{\leq z} \rightarrow K^{\bar{L}_{z}}\left(\widetilde{\mathfrak{N}}_{z}\right)$ be the composition across the top row in (3.5). Then $f_{1}=i_{z}^{*} \operatorname{res}_{z} r_{z}^{*} \varphi_{z}$, where $i_{z}^{*}, \operatorname{res}_{z}$, and $\varphi_{z}$ are isomorphisms and $r_{z}^{*}$ is surjective. If $y<z$, then $\varphi_{w}\left(\mathcal{H}_{y}\right) \subseteq K^{\bar{G}}\left(Z_{\leq y}\right)$ and so it follows from the exactness of the top row of diagram (3.4) that $\varphi_{z}\left(\mathcal{H}_{y}\right)$ is in the kernel of $r_{z}^{*}$. Therefore, $\mathcal{H}_{y}$ is in the kernel of $f_{1}$.

By Theorem $8.3(2), r_{z}^{*} \varphi_{z}\left(\theta_{\lambda} T_{z}\right)=\epsilon_{z} q_{z, 1}^{*}\left(\left[\mathcal{L}_{\lambda}\right]\right)$, and so

$$
f_{1}\left(\theta_{\lambda} T_{z}\right)=\epsilon_{z} i_{z}^{*} \operatorname{res}_{z} q_{z, 1}^{*}\left(\left[\mathcal{L}_{\lambda}\right]\right)
$$


Let $g_{z}: F \rightarrow Z_{z}$ be the inclusion and let $f_{z}: L_{z} / B_{z} \rightarrow G / B$ be the canonical embedding. Then the diagram

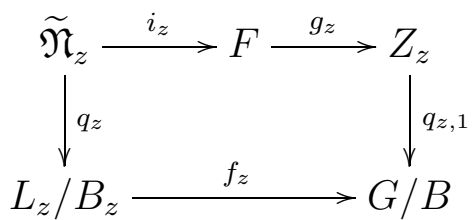

commutes. The restriction map $\operatorname{res}_{z}: K^{\bar{G}}\left(Z_{z}\right) \rightarrow K^{\bar{L}_{z}}(F)$ is induced by the functor $g_{z}^{*}$ from $\bar{G}$-equivariant coherent sheaves on $Z_{z}$ to $\bar{L}_{z}$-equivariant sheaves on $F$. Therefore,

$$
i_{z}^{*} \operatorname{res}_{z} q_{z, 1}^{*}\left(\left[\mathcal{L}_{\lambda}\right]\right)=\left[i_{z}^{*} g_{z}^{*} q_{z, 1}^{*}\left(\mathcal{L}_{\lambda}\right)\right]=\left[q_{z}^{*} f_{z}^{*}\left(\mathcal{L}_{\lambda}\right)\right]=\left[q_{z}^{*}\left(\mathcal{L}_{\lambda}^{z}\right)\right]=q_{z}^{*}\left[\mathcal{L}_{\lambda}^{z}\right]
$$

and so $f_{1}\left(\theta_{\lambda} T_{z}\right)=\epsilon_{z} q_{z}^{*}\left[\mathcal{L}_{\lambda}^{z}\right]$.

To prove Theorem $3.8(3)$, let $f_{2}: \mathcal{H}_{\leq z}^{I J} \rightarrow K^{\bar{L}_{z}}\left(\mathfrak{N}_{z}\right)$ be the composition across the bottom row in (3.5). Then $f_{2}=\left(i_{z}^{I J}\right)^{*} \operatorname{res}_{z}^{I J}\left(r_{z}^{I J}\right)^{*} \psi_{z}$ and $f_{2} \chi^{\leq z}=\left(p_{z}\right)_{*} f_{1}$. If $y$ is in $W^{I J}$ and $y<z$, then $\psi_{z}\left(\mathcal{H}_{y}^{I J}\right) \subseteq K^{\bar{G}}\left(X_{\leq y}^{I J}\right)$ and so it follows from the exact sequence (3.1) that $\psi_{z}\left(\mathcal{H}_{y}^{I J}\right)$ is in the kernel of $\left(r_{z}^{I J}\right)^{*}$. Therefore, $\mathcal{H}_{y}^{I J}$ is in the kernel of $f_{2}$. Suppose $\lambda$ is in $X(T)$. Then

$$
f_{2}\left(C_{w_{I}} \theta_{\lambda} T_{z} C_{w_{J}}\right)=f_{2} \chi^{\leq z}\left(\theta_{\lambda} T_{z}\right)=\left(p_{z}\right)_{*} f_{1}\left(\theta_{\lambda} T_{z}\right)=\epsilon_{z}\left(p_{z}\right)_{*} q_{z}^{*}\left(\left[\mathcal{L}_{\lambda}^{z}\right]\right) .
$$

As observed before the statement of Theorem 3.8, $\left\{C_{w_{I}} \theta_{\lambda} T_{z} C_{w_{J}} \mid \lambda \in X(T)_{z}^{+}\right\}$is a $A$-basis of $\mathcal{H}_{z}^{I J}$, and by Lemma 2.2, $\left\{\epsilon_{z}\left(p_{z}\right)_{*} q_{z}^{*}\left(\left[\mathcal{L}_{\lambda}^{z}\right]\right) \mid \lambda \in X(T)_{z}^{+}\right\}$is an $A$-basis of $K^{\bar{L}_{z}}\left(\mathfrak{N}_{z}\right)$. It follows that $f_{2}$ is an isomorphism.

Finally, to prove Theorem 3.8 (1) it remains to show that $\eta_{*}^{\leq z}$ is surjective and that $\psi_{z}$ is an isomorphism. The fact that $\eta_{*}^{\leq z}$ is surjective was established in Corollary 3.5. Since $\psi_{z} \chi^{\leq z}=\eta_{*}^{\leq z} \varphi_{z}$, we see that $\psi_{z}$ is a surjection as well. Induction on the length of $z$ and statement (3) of the theorem show that

$$
\left\{\psi_{z}\left(C_{w_{I}} \theta_{\lambda} T_{y} C_{w_{J}}\right) \mid y \in W^{I J}, y \leq z, \lambda \in X(T)_{y}^{+}\right\}
$$

is a linearly independent subset of $K^{\bar{G}}\left(X_{\leq z}^{I J}\right)$ and so $\psi_{z}$ is an injection. This completes the proof of Theorem 3.8.

Acknowledgments: This work was partially supported by a grant from the Simons Foundation (Grant \#245399 to J. Matthew Douglass). The authors acknowledge the financial support of a DFG grant for the enhancement of bilateral cooperation and the DFG-priority program SPP1388 "Representation Theory." The authors would like to thank Victor Ostrik for clarifying the assertions in [17], William Graham and an anonymous referee for help with the proof of Proposition 6.2, and the same anonymous referee for numerous suggestions that substantially improved the paper.

\section{REFERENCES}

[1] B. Broer, Line bundles on the cotangent bundle of the flag variety, Invent. Math. 113 (1993), 1-20. 7

[2] N. Chriss and V. Ginzburg, Representation theory and complex geometry. Birkhäuser, Boston, 1997. 2, 3, 6, 24 
[3] C.W. Curtis, On Lusztig's isomorphism theorem for Hecke algebras, J. Algebra 92 (1985), 348365. 1

[4] C.W. Curtis, N. Iwahori, and R. Kilmoyer, Hecke algebras and characters of parabolic type of finite groups with $(B, N)$-pairs, Inst. Hautes Études Sci. Publ. Math. 40 (1971), 81-116. 1

[5] C.W. Curtis and I. Reiner, Methods of representation theory. Vol. II. With applications to finite groups and orders. Pure and Applied Mathematics. John Wiley \& Sons, New York, 1987. 7, 10, 12,23

[6] J.M. Douglass, An inversion formula for relative Kazhdan-Lusztig polynomials, Comm. in Algebra 18 (1990), 371-387. 10, 15

[7] J.M. Douglass and G. Röhrle, The geometry of generalized Steinberg varieties, Adv. Math. 187 (2004), 396-416. 1, 4, 6, 10

[8] J.M. Douglass and G. Röhrle, Homology of generalized Steinberg varieties and Weyl group invariants, Trans. Amer. Math. Soc. 360 (2008), 5959-5998. 2

[9] J.M. Douglass and G. Röhrle, The Steinberg variety and representations of reductive groups, J. Algebra 321 (2009), 3158-3196. 2

[10] N. Iwahori, On the structure of a Hecke ring of a Chevalley group over a finite field, J. Fac. Sci. Univ. Tokyo Sect. I 10 (1964), 215-236. 1

[11] D. Kazhdan and G. Lusztig, Representations of Coxeter groups and Hecke algebras, Invent. Math. 53 (1979), 165-184. 4, 5, 15

[12] D. Kazhdan and G. Lusztig, Proof of the Deligne-Langlands conjecture for Hecke algebras, Invent. Math. 87 (1987), 153-215. 1, 2, 11, 24

[13] G. Lusztig, On a theorem of Benson and Curtis, J. Algebra 71 (1981), 490-498. 1

[14] G. Lusztig, Singularities, character formulas, and a q-analog of weight multiplicities, in Analysis and topology on singular spaces, II, III (Luminy, 1981), Astérisque, vol. 101, Soc. Math. France, Paris, 1983, 208-229. 5

[15] G. Lusztig, Bases in equivariant K-theory, Represent. Theory 2 (1998), 298-369. 2, 5, 6, 7, 9, $15,16,17,21,26,27$

[16] K. Nelsen and A. Ram, Kostka-Foulkes polynomials and Macdonald spherical functions, in Surveys in combinatorics, 2003 (Bangor), London Math. Soc. Lecture Note Ser., vol. 307, Cambridge Univ. Press, Cambridge, 2003, 325-370. 21, 22

[17] V. Ostrik, On the equivariant K-theory of the nilpotent cone, Represent. Theory 4 (2000), 296-305. 2, 7, 28

[18] W. Soergel, Kazhdan-Lusztig polynomials and a combinatoric[s] for tilting modules, Represent. Theory 1 (1997), 83-114. 15

[19] R.W. Thomason, Algebraic K-theory of group scheme actions, in Algebraic topology and algebraic $K$-theory (Princeton, N.J., 1983), Ann. of Math. Stud., vol. 113, Princeton Univ. Press, Princeton, NJ, 1987, 539-563. 3

Department of Mathematics, University of North Texas, Denton TX, USA 76203

E-mail address: douglass@unt.edu

$U R L:$ http://hilbert.math.unt.edu

Fakultät für Mathematik, Ruhr-Universität Bochum, D-44780 Bochum, Germany

E-mail address: gerhard.roehrle@rub.de

URL: http://www.ruhr-uni-bochum.de/ffm/Lehrstuehle/Lehrstuhl-VI 